\title{
The Origin of Cosmic Structures Part 1-Stars to Superclusters
}

\author{
J. C. Botke \\ Nogales, Arizona, USA \\ Email: jcbotke@gmail.com
}

How to cite this paper: Botke, J.C. (2021) The Origin of Cosmic Structures Part 1Stars to Superclusters. Journal of High Energy Physics, Gravitation and Cosmology, 7, 1373-1409.

https://doi.org/10.4236/jhepgc.2021.74085

Received: August 10, 2021

Accepted: October 16, 2021

Published: October 19, 2021

Copyright ( 2021 by author(s) and Scientific Research Publishing Inc. This work is licensed under the Creative Commons Attribution International License (CC BY 4.0).

http://creativecommons.org/licenses/by/4.0/

\begin{abstract}
Our original intent was to explain the origin of large HI structures. In order to understand HI structures, however, it is first necessary to understand the origin of both galaxies and galaxy clusters. Explaining their origin is the purpose of Part 1 of this work. In our new model of cosmology, the creation of protons during nucleosynthesis was regulated by an imprint embedded in the vacuum in a manner that eventually resulted in the cosmic structures we now observe. Immediately after nucleosynthesis and for a considerable period afterward, the evolution was dominated by the expansion of the universe. Gradually, gravitational influences became important until eventually, the two became equal. At that point, the structures ceased to increase in size, and thereafter, their evolution was dominated by the gravitational interaction of the particles. The zero-velocity point for galaxies and galaxy clusters occurred at the usually accepted time of the beginning of galaxy formation. The initial population of stars also started their compaction at that same time but, in this case, partially for reasons having to do with the temperature of the proton gas. Many details of the evolution of the structure are discussed. We discuss the equilibrium of galaxy clusters and present a model that can potentially account for the present-day energy of the intracluster gas. Another outcome is that, at the time $t_{G}$ when the galaxies reached their zero-velocity point, they were several times larger than their present-day size, a fact that is critical for understanding the origin of the larger HI rings. In Part 2 of this work, we show that the HI structures can readily be understood in terms of the model developed here.
\end{abstract}

\section{Keywords}

HI rings, Galaxy Evolution, Galaxy Cluster Evolution, Stellar Evolution, Nucleosynthesis, Early Universe, Time-Varying Curvature 


\section{Introduction}

Our original concept was a paper limited to the problem of the HI rings but understanding rings requires an understanding of the origin of everything else, especially of galaxies and galaxy clusters so, as sometimes happens, a single paper of limited scope has grown into two papers covering a wider range of topics. Part 1 deals with the origin of cosmic structures in general. Part 2 then builds on this background to explain the origin of the HI rings. All this work is based on the new cosmology described in [1].

We will begin with the stars. Star formation has been and is going on throughout the universe and the processes involved are generally well understood. What these new stars all have in common is that they are in one form or another the result of the recycling of stellar debris from previous generations of stars. Those remnants not only provided the needed material but also seeded the star formation regions with focal points about which accretion could begin. Clearly, however, the process of recycling can only be carried back so far and eventually we come to the problem of accounting for the first generation of stars.

\section{Start(ing) from Scratch}

A common viewpoint is that the distribution of matter resulting from nucleosynthesis was uniform but if we assume that to be the case, we immediately run into significant problems. The first is that with a uniform distribution of matter, not only would there not have been any star formation focal points, we showed in [1] that the formation of such points by random density fluctuations is impossible.

The second problem is the extreme mass of stars relative to the average background density of matter. In terms of present-day values, the average density of baryons in the universe is on the order of $1 \mathrm{~m}^{-3}$. Building a star out of such a background would require a volume of space roughly equal to the size of a dwarf galaxy and dwarf galaxies contain $10^{8}-10^{9}$ stars rather than just one. If instead, we use a value of $10^{6} \mathrm{~m}^{-3}$ which is typical of the present-day average density of the Milky Way, we find that building a star would require a volume equal to about $20 \%$ the size of a globular cluster. Either way, random accretion out of the background just isn't going to work because organizing accretion over such vast distances, even after accounting for the expansion of the universe, would be impossible and this is leaving aside the fact that millions of stars would have been competing for the same background.

Another way to look at this is that the disparity between the organization of the matter in the universe on all scales and the average background density is just too great. Observation tells us that the universe is highly organized on scales ranging from the size of stars to superclusters and it should be clear that no sequence of random events could ever result in such colossal organization. Even if we managed to create the requisite number of stars for a galaxy, we would still be faced with the equally impossible problem of organizing them into those galax- 
ies. The conclusion is that some organizing principle must have been in play from the very beginning and in [1], we proposed a new model of cosmology that expresses that idea.

We will now take up the story at the time of recombination and work backward to a Plank era inflation that started the whole universe going. In Section 15 of [1], we proved that superclusters are responsible for the large peaks of the $\mathrm{CMB}$ spectrum. That being the case, it immediately follows that superclusters in some form must have been in existence at that time which in turn means that they must have come into existence at some earlier time. We also know that at the time of recombination, superclusters, and hence the CMB spectrum peaks, were vastly too large to be explained by any causal process. (It is this consideration that proves that the acoustic oscillation model of the CMB peaks is just nonsense.) Moving backward in time, since nothing much happened between the time of recombination and nucleosynthesis, it is natural to suppose that the material manifestation of superclusters originated during nucleosynthesis. But at that time, causality was even more restrictive so we must look back even further in the hopes of finding a way out of the causality problem.

We will pause our time travels for a moment to outline the new model of nucleosynthesis. Observations of the densities of a few light elements can carry us back to the termination of nucleosynthesis proper (at a time of about $4000 \mathrm{~s}$ ). Working backward again using known nuclear reactions, we discover that nucleosynthesis proper began with a mix of about $80 \%$ protons and $20 \%$ neutrons. That gets us back to about $t \approx 10^{-5} \mathrm{~s}$ but that is as far as observation can take us. The real problem of nucleosynthesis, then, is to account for the original mix of protons and neutrons. The standard model asserts that the process began much earlier with radiation which via various reactions described by field theory resulted in the final mix of protons and neutrons. There is, however, no evidence to support that viewpoint. Three of the bigger problems with such a model are that it violates causality, is too complex, and cannot explain the matter/antimatter asymmetry of the universe. We assert that such a beginning never happened and, in its place, we proposed a much simpler model that does not suffer from these limitations.

The idea is that there was no existence before the onset of nucleosynthesis other than the vacuum and matter came into existence at a time of about $10^{-5} \mathrm{~s}$ via a process by which roughly $0.1 \%$ of the total vacuum energy was converted into neutron/antineutron pairs. From that beginning, a combination of $\beta$ decay and cascades of neutrino reactions resulted in the production of protons. Simultaneously, pair annihilation produced the radiation that became the CMB.

All this would have eventually resulted in nothing but radiation except for a very small bias in the pair production process. An average bias of $2-4$ extra neutrons for every $10^{8}$ pairs is sufficient to account for both the $100 \%$ matter/antimatter asymmetry of the universe and the total amount of matter in existence at the present time. 
Getting back to the superclusters, at the time of nucleosynthesis the causality problem had not only gotten worse concerning superclusters, but it had also become a problem concerning all the other cosmic structures from galaxy clusters on down. And yet, these must have been there at that time because superclusters are nothing more than a collection of galaxy clusters that contain collections of galaxies and so on.

The final jump backward is to a Plank era inflation. One critical attribute of the vacuum during the Plank era was that time, distance, and energy were uncertain. To see this, imagine that we are interested in measuring the duration of some event. To do so, we need a clock whose ticks are of a shorter duration than that of the event so to measure events of shorter and shorter duration, we must keep subdividing the ticks. We eventually come to the point at which our tick is on the order of the Plank time. We now assert that the Plank tick cannot be subdivided and as a result, time becomes uncertain. The same idea holds for distance and energy as well. (In different contexts, many people have developed models based on this idea in attempts to tame the infinities of field theory so the idea of a Plank limit is not new.) An important consequence of this uncertainty is that causality had no meaning since neither time nor distance had any exact meaning. It is here, then. during the Plank inflation that we are finally able to escape the constraints imposed by causality (and entropy). In [1], we showed that the initial expansion of the universe was exponential and lasted until the age of the universe became large compared to the Plank time uncertainty. From that point onwards, the evolution of the universe could be described by Einstein's equations with the understanding that Einstein's equations have validity only for dimensions large compared to Plank dimensions.

That idea by itself, however, does not explain superclusters. To make the final step, structure is needed and we conclude that large smooth acausal vacuum structures or imprints came into existence during the inflation with sizes ranging from the size of stars up to the size of the inflating universe. Normally, systems do not spontaneously evolve into a highly organized state starting from a highly disorganized state but this is what actually happened and for it to have happened, there must have been an absence of causality.

We now return to the time of nucleosynthesis. At that time, those smooth imprints on the vacuum regulated the pair production process in such a manner as to create all the comic structures we now see.

We presented some evidence for this idea in [1] and it will become apparent in Part 2 that these same imprints are responsible for the observed HI structures. All cosmic structures follow a general plan as demonstrated by the observed distributions of their counts and sizes, [2]. We found that these imprints exhibit a fractal dimension of 1.1 which is consistent with the nearly 1-dimensional, filament manifestation of matter in the universe that we observe. It is also clear that there is considerable variation in the details of the structures since galaxies, for example, have a range of morphologies, sizes, and stellar masses. They all came into existence with roughly their final mass and basic morphology and while 
compaction played a major role in the evolution of the structures, the original size of the structures was set by the imprint.

Additional evidence for this model follows from the discovery of the galaxy, ZN-z11 [3] with a redshift of $z=11.1$. (The reference quotes a lookback time of $\sim 400 \mathrm{Myr}$ but the relationship between redshift and lookback time is model dependent and using the results of the new model, we find a lookback time of $\sim 334$ Myr.) This galaxy has a mass of $\sim 10^{9} M_{\odot}$ and is unexpectedly luminous indicating a much higher than expected star formation rate. From the point of view of the new model, however, this is not remarkable at all because the galaxy with some percentage of its stars came into existence in proto form at the time of nucleosynthesis with the actual initial star formation occurring simultaneously throughout the galaxy at a time beginning at $t=t_{G}$ where the latter is the usually accepted time of the beginning of galaxy formation.

Returning now to the original question that started this discussion, the universe did not begin with a uniform distribution of matter. Instead, it began with an inflation during which a complete blueprint of all the cosmic structures was imprinted on the vacuum. Over time, compactions and interactions continued to modify the details but, on the whole, such processes were of secondary importance relative to the original definitions of the structures.

\section{Vacuum Energy is the Reality of Dark Matter}

We noted earlier that the basis of nucleosynthesis was a conversion of a small percentage of the vacuum energy into neutron/antineutron pairs. More than $99 \%$ of this energy remained in the vacuum, however, and that energy has important consequences for cosmology. Its existence means that it must appear in any expression of Einstein's equations as a contribution to the energy-momentum tensor and it is this contribution that is responsible for the phenomena attributed to dark matter and dark energy. It happens, for example, that the new model prediction for the present-day background vacuum energy density is within a factor of 3 of the generally assumed density of dark energy.

Another prediction follows from the form of Einstein's equations for a rotating galaxy. The particular example we will consider is a stationary axisymmetric disk. The metric and the equations are given in [1] where the notation is explained. Since the vacuum energy responds to the curvature of space in the same way as does ordinary matter, the geodetic equations apply to each small volume of the vacuum. Thus,

$$
\frac{\mathrm{d} \bar{v}^{\mu}}{\mathrm{d} \tau}+\Gamma_{v \sigma}^{\mu} \bar{v}^{v} \bar{v}^{\sigma}=0
$$

where $\bar{v}^{\mu}$ is the dimensionless 4 -velocity of the vacuum. The first two of these equations vanish identically. The last two are given by,

$$
\begin{aligned}
& E\left(-A^{(0,1)}+l^{2}\left(\dot{\phi}_{v a c}-\bar{\omega}\right)^{2} B^{(0,1)}\right)+D\left(A^{(1,0)}-l^{2}\left(\dot{\phi}_{v a c}-\bar{\omega}\right)^{2} B^{(1,0)}\right) \\
& -2 l B\left(\dot{\phi}_{v a c}-\bar{\omega}\right)\left(I E \bar{\omega}^{(0,1)}+D\left(\dot{\phi}_{v a c}-\bar{\omega}-l \bar{\omega}^{(1,0)}\right)\right)=0
\end{aligned}
$$




$$
\begin{aligned}
& C\left(A^{(0,1)}-l^{2}\left(\dot{\phi}_{v a c}-\bar{\omega}\right)^{2} B^{(0,1)}\right)+E\left(-A^{(1,0)}+l^{2}\left(\dot{\phi}_{v a c}-\bar{\omega}\right)^{2} B^{(1,0)}\right) \\
& +2 l B\left(\dot{\phi}_{v a c}-\bar{\omega}\right)\left(l C \bar{\omega}^{(0,1)}+E\left(\dot{\phi}_{v a c}-\bar{\omega}-l \bar{\omega}^{(1,0)}\right)\right)=0
\end{aligned}
$$

The arguments $(l, z)$ of the functions have been suppressed. Since both $\dot{\phi}_{v a c}$ and $\bar{\omega}$ are small, we expect the equations to be satisfied in the limit that they vanish. The resulting equations have the solution $A^{(0,1)}=A^{(1,0)}=0$ which is consistent with the metric becoming the flat metric in that limit. After substituting, we find that $\dot{\phi}_{v a c}=\bar{\omega}$ solves both of the equations. Computing the norm of the velocity gives

$$
\bar{v}^{\mu} \bar{v}_{\mu}=-1=-A+B l^{2}\left(\dot{\phi}_{v a c}-\bar{\omega}\right)^{2}=-A
$$

so, we have the result, $A=1$.

What we find is that the vacuum is rotating. To better understand what is happening, we consider the usual inertial frame dragging argument starting with the fact that the covariant component of the angular momentum, $p_{\phi}$, is conserved on a geodetic. Raising the index gives

$$
\begin{aligned}
& p^{t}=g^{t t} p_{t}+g^{t \phi} p_{\phi} \\
& p^{\phi}=g^{\phi t} p_{t}+g^{\phi \phi} p_{\phi}
\end{aligned}
$$

If we now assume that the vacuum angular momentum $p_{\phi}=0$, we eventually obtain the result that

$$
\dot{\phi}_{v a c} \equiv \frac{\mathrm{d} \phi}{\mathrm{d} c t}=\frac{p^{\phi}}{p^{t}}=\frac{g^{\phi t}}{g^{t t}}=\bar{\omega}
$$

which is the result we had just found. The conclusion is that the vacuum is rotating at the overall rotation rate of the galaxy and is doing so with zero angular momentum.

We now consider the galactic matter whose velocity must also satisfy the same geodetic equations. In this case, there is no reason to suppose that the total angular momentum vanishes so we separated the angular velocity into a component with vanishing angular momentum and a residual with non-vanishing angular momentum,

$$
\dot{\phi}_{m}=\dot{\phi}_{m, r}+\bar{\omega} \text {. }
$$

The residual angular velocity is then,

$$
\dot{\phi}_{m, r}(l, z)=\frac{v}{l}-\bar{\omega}(l, z)
$$

where $v=\bar{\omega}\left(l_{G}, 0\right)$ is the observed constant stellar velocity. Thus, $\dot{\phi}_{m, r}\left(l_{G}, 0\right)=0$ which means that the outermost stars are at rest in a rotating vacuum. This fact greatly reduces the effective mass necessary to achieve the flat velocity spectrum.

The vacuum energy density model also solves the problem of explaining how just the right amount of dark matter always manages to accumulate just outside every galaxy and why we don't find the odd galaxy here and there that is missing 
its dark matter halo.

In later sections of this paper, we will discuss additional instances in which vacuum energy readily accounts for phenomena attributed to dark matter.

Dark matter does not exist.

\section{Origin of Rotation}

In [1], we didn't give much thought to the problem of where galaxy rotation, for example, originated. We noted earlier that the distribution of matter in the universe is highly organized; in fact, vastly too organized on all scales to be a consequence of any causal physical process, random or otherwise. Thinking a moment about galaxies, we will find a similarly high degree of organization is characteristic of their rotations and in fact, of all structures within and outside galaxies. Galaxies are for the most part independent entities with a broad range of dimensions and morphologies so without some organizing principle, we would expect the observed rotations to be spread over a broad spectrum. Instead, the Tully-Fisher relationship gives evidence that their rotations are to some extent coordinated as does the fact that they all are observed to have flat velocity profiles. The same can be said of the HI outer rings which are discussed in Part 2.

Referring now to galaxy clusters, only about $25 \%$ [4] of clusters exhibit rotation but given their size, the surprising thing is that any clusters are rotating. As we will show later, because of causality constraints, they were well on in their evolution before signals could traverse the dimensions of the clusters and by that time, no gravitational process could have gotten the whole thing rotating.

All this indicates that the rotation is primordial but that immediately brings up another problem. The authors of [4] report rotation velocities on the order of a few hundred $\mathrm{km} \cdot \mathrm{s}^{-1}$ which, interestingly, is of the same order of magnitude as the velocity of stars in galaxies. It is well known that particle velocity varies inversely with the scaling so such present-day velocities would imply velocities on the order of $10^{12} \mathrm{~ms}^{-1}$ at the time of nucleosynthesis which is clearly impossible. This, in turn, means that a primordial rotation expressed in terms of an orbital motion of particles is impossible.

Since the rotation cannot be explained in terms of particle motion, it must be the vacuum that is rotating and it is not a big leap to suppose that on all length scales, each region of the Plank era imprint was rotating. Thus, on the larger scales, the rotation is not a matter of particle motion but of vacuum rotation.

\section{Cosmic Structures}

The filament structure that defines superclusters was fixed by the imprint and because they are vastly too large to be subject to gravitational interaction when taken as a whole, they are today much the same as they were initially. Components within the superclusters such as galaxy clusters certainly do evolve but the grand filaments do not.

To study the evolution of clusters, galaxies, and stars, we consider the motion 
of a particle at the outer edge of some volume containing the mass of the structure. The particle's initial velocity is given by the expansion of the universe and its acceleration has two components; namely the inward acceleration due to the enclosed mass and outward acceleration due to the expansion. The coordinates are illustrated in Figure 1.

The present-day size of any particular structure is denoted by $R_{0}$ and the scaling is given by $a(t)$. The parameter $f(t)=R(t) / R_{0}$ is the ratio of the particle distance at any time to $R_{0}$. The black lines indicate the paths that objects would follow in response to just the scaling. On these paths, $f$ is constant. The outer value, $f_{\text {Sur }}$, is the value that encloses the volume of the background particle density necessary to match the total mass of the structure. The upward bend of the scaling curves indicates the acceleration of the scaling predicted by the new model. The red line is a possible path of the outer surface of the structure.

Clearly, this model on its own is only designed to describe the evolution of structures up to the point at which the radial velocity vanishes. Before that time, structure evolution was largely a consequence of the expansion of the universe with the total mass of the structure gradually exerting its influence. After the zero-velocity point (ZVP) is reached, the details of the structure become important and this model has nothing to say about that. For example, if the structure virializes at that point, then the actual curve would thereafter be flat. In other cases, it might increase again initially and then later approach the present-day size from above. Causality plays a role in this as well and it will be discussed at the appropriate time.

The acceleration of a particle at the outer edge of the structure mass is given by

$$
\ddot{R}(t)=-\frac{G M_{\text {eff }}(t)}{R(t)^{2}}+R_{0} f_{s} \frac{\ddot{a}(t)}{a_{0}}
$$

for a particle with an initial value of $f_{\text {starting }}=f_{s}$. The scaling predicted by the new model is given by

$$
a(t)=a_{*}\left(\frac{t}{t_{0}}\right)^{\gamma_{*}} \mathrm{e}^{c_{1} t / t_{0}}
$$

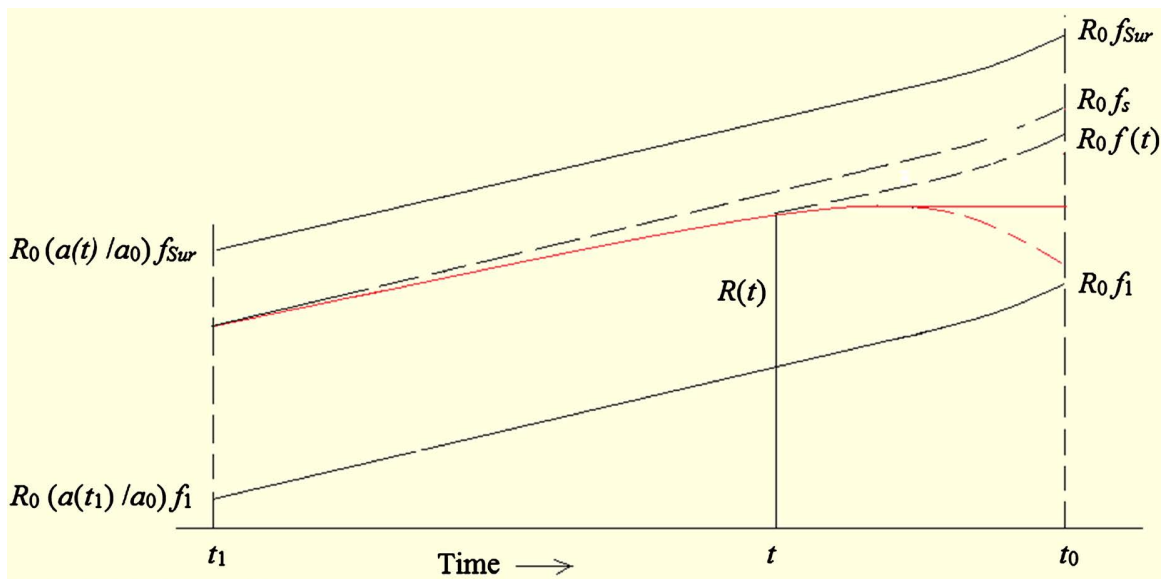

Figure 1. Accretion model coordinates. 
where $\gamma_{*}=0.5, c_{1}=0.49$, and $a_{*}=a_{0} \mathrm{e}^{-c_{1}}$. This is an exact solution of Einstein's for a metric with time-varying curvature. The value of $c_{1}$ corresponds to a Hubble value of $H_{0}=70$. (It is a little larger than the value used in [1]. The adjustment was made because a value of 70 gives a better fit to the luminosity distance data.) After taking the derivatives and substituting, we have

$$
\ddot{R}(t)=-\frac{G M_{\text {eff }}(t)}{R(t)^{2}}+R_{0} f_{s}\left(-\gamma_{*}+\left(\gamma_{*}+c_{1} \frac{t}{t_{0}}\right)^{2}\right) \frac{a(t)}{a_{0}} \frac{1}{t^{2}} .
$$

Note that the scaling contribution is negative for times earlier than $t / t_{0}=0.42$ and positive thereafter.

Three issues need to be handled. The first has to do with the enclosed mass of the cluster. If we consider a particle located at the radius of the volume needed to form the cluster from the background density, the particle density is the same everywhere because that has not yet been any compaction defining the structure. This means that at that point, we must have $M_{\text {eff }}\left(t_{1}\right)=0$ so the particle will experience only the scaling. The mass of the structure is there but it is spread out at the background density. After the structure mass has undergone some compaction, the surface will have moved towards the center but we must continue to allow for the background density of particles. We can do this by defining the effective mass to be the difference between the actual structure mass within the evolving outer surface and the mass of the same volume filled at the background density. With a little algebra, we find that,

$$
M_{\text {eff }}(t)=M_{V C}\left(1-\left(\frac{R(t)}{R_{\text {Sur }, s}(t)}\right)^{3}\right)
$$

The variable $R_{\text {Sur,s }}(t)$ is the distance from the surface to the outer scaling-only curve shown in the figure. This effective mass vanishes for a particle at the outer curve and has a value of very close to the total structure mass when the structure reaches its present-day size.

The second issue is to somehow introduce the notion of a perturbation to get things going. We accomplish this by means of the parameter, $f_{s}$ which fixes the starting position of the outer rim. If we initialize $f_{s}$ with the value $f_{\text {sur }}$, the effective mass vanished so there is no compaction. If we chose any smaller value, the effective mass is not zero thus giving the structure initial compaction which will start the evolution.

The last issue concerns the paths calculated for individual galaxies in the case of the cluster model. Galaxies outside the boundary of the cluster experience the full effective mass. Those inside the boundary experience that portion of the effective mass that is inside their position.

We now integrate (5-3) using the $4^{\text {th }}$ order Runge-Kutta method. We specify the initial position of the particle using $f_{s}$ and assume that the initial velocity is given by $\dot{a}\left(t_{4000}\right)$. The latter is completely reasonable because initially the particles are rapidly moving apart from each other as a result of the expansion 
and have no collective interaction because of the limitations imposed by causality.

We will repeatedly refer to the time of nucleosynthesis by which we mean $4000 \mathrm{~s}$. We will also generally refer to densities in terms of their present-day values with the understanding that the actual densities at earlier times were much larger. For example, a present-day particle density of $1 \mathrm{~m}^{-3}$ corresponds to a value of $4.3 \times 10^{21} \mathrm{~m}^{-3}$ at the time of nucleosynthesis.

We will begin with galaxy clusters and work out way down to the stars.

\section{Galaxy Clusters}

For these calculations, we will take our dimensions from those of the Virgo cluster. While the details of the results are dependent on these values, the general character of the results is not so the results are generally applicable to other clusters.

There are uncertainties in the values of the relevant parameters and the values are not entirely consistent. Researchers generally accept that the distance to the cluster is $16.5 \mathrm{mpc}$ and that the average radius is $2.2 \mathrm{mpc}$. It is also generally agreed that the average particle density of the intracluster medium (ICM) is $10^{3}$ $\mathrm{m}^{-3}$. The generally accepted total particle mass, however, is not consistent with those values.

If we compute the mass using the average density, the result is $M \approx 2 \times 10^{45} \mathrm{~kg}$ which is about $82 \%$ of the generally accepted total mass including dark matter, $1.2 \times 10^{15} M_{\odot}=2.45 \times 10^{45} \mathrm{~kg}$, [5]. As we will see, however, this is too large a value. Instead, a value of $M_{C} \approx 1 \times 10^{45} \mathrm{~kg}$ appears to be closer to reality. If the accepted value of the total particle mass, $3 \times 10^{14} M_{\odot}=6 \times 10^{44} \mathrm{~kg}$ is correct, the difference is a deficit of $4 \times 10^{44} \mathrm{~kg}$. One of our assertions is that vacuum energy accounts for the phenomena attributed to dark matter. In this case, we will show that the vacuum can easily account for this deficit mass. On the other hand, if the particles make up the whole of the total mass, then the vacuum contribution is minimal. Either way, there is no dark matter. In what follows, we will use a base value of $M_{C}=1 \times 10^{45} \mathrm{~kg}$ but, for comparison, will also show results using $M_{C}=2 \times 10^{45} \mathrm{~kg}$.

We will first establish that the standard accretion model of cluster formation initiated by small density perturbations in the early universe is unworkable and then show that clusters can be understood in terms of the new model of nucleosynthesis. An important point is that with accretion starting from the average background density of $1 \mathrm{~m}^{-3}$, the initial volume necessary to create the cluster must have been about 10 times the size of the cluster. Another important point that will come up later is that the model is only concerned with the total mass and not how that mass happens to be configured.

According to the accretion model, clusters could not have come into existence until there was something to cluster. In Figure 2, we show the model calculation results for a starting time of $t_{G}=3 \times 10^{16} \mathrm{~s}$.

We don't show it but with $f_{s}=10$, the particle would follow the outer surface 
(blue) scaling-only curve. From the figure, we find that a value of $f_{s}<7$ is necessary before the accretion model has any chance of accounting for even the existence of the cluster and if we imagine that the cluster virializes at the ZVP, then a value of $f_{s} \approx 4$ is necessary. The conclusion is that with a starting time of $t=t_{G}$ and anything less than an initial compaction ratio of around 2.5 , accretion cannot account for the present-day size of the cluster and 2.5 is certainly far beyond what would anyone would consider a small perturbation.

We next consider the problem of capturing objects from outside the surface of the cluster mass. The results are shown in Figure 3.

We find that galaxy capture is only possible out to distances a little bit greater than $f_{G}=6$.

No matter the details, what we have learned is that for accretion to work, the cluster must have been a well-defined structure at the time $t=t_{G}$ and this, in turn, implies that it must have come into existence at a much earlier time. Since we have argued from the beginning that all structures were formed during nucleosynthesis with more or less their final mass, we will calculate the evolution starting at a time of $t=4000 \mathrm{~s}$. The result is shown in Figure 4.

The initial compaction ratio is 1.36 . We just used the phrase, initial compaction, but that really isn't the correct term because the protons making up the

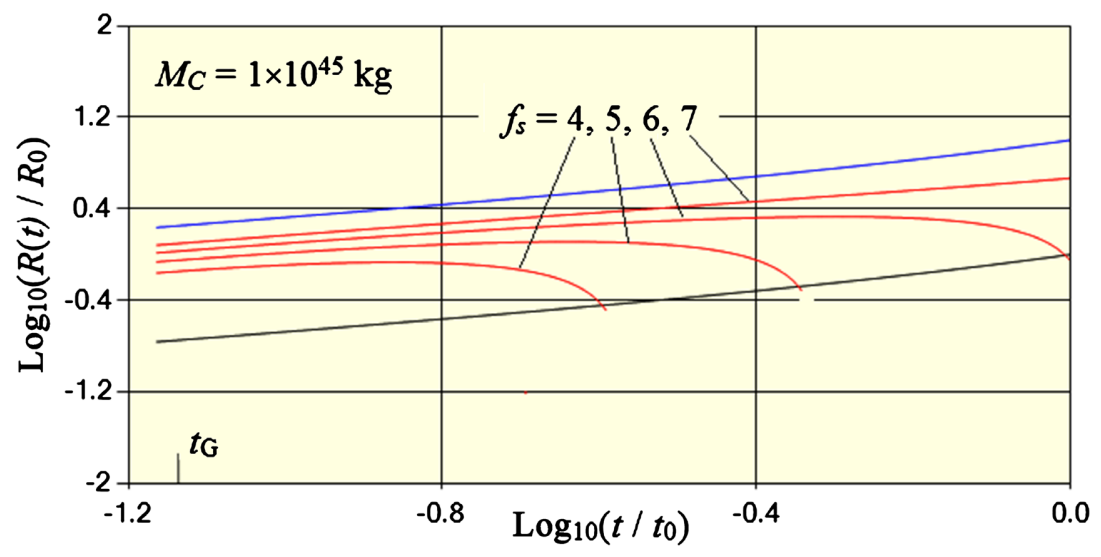

Figure 2. Solution for 4 values of the starting position, $f_{s}$ with $t_{s}=t_{G}=3 \times 10^{16} \mathrm{~s}$.

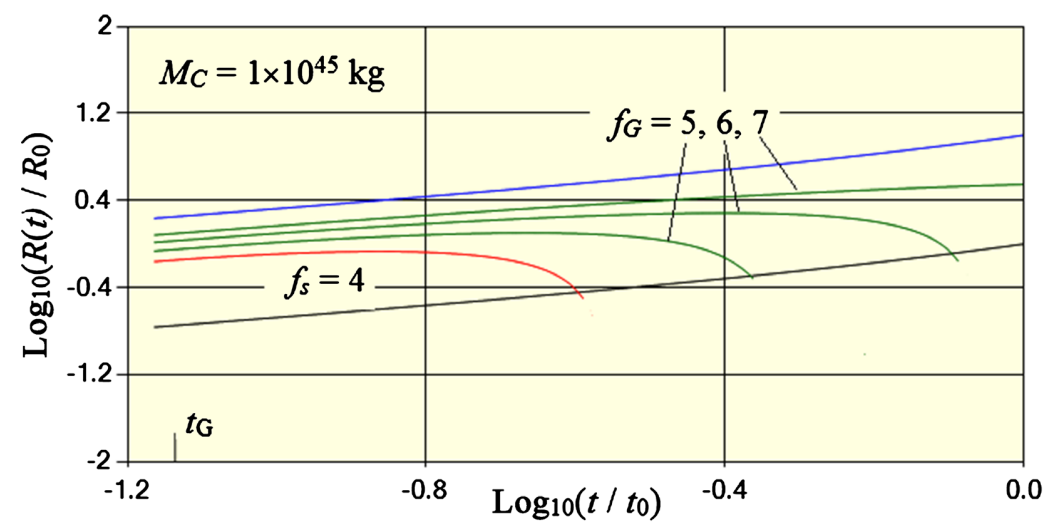

Figure 3. Model solution including 3 possible external galaxies with $f_{s}=4$. 


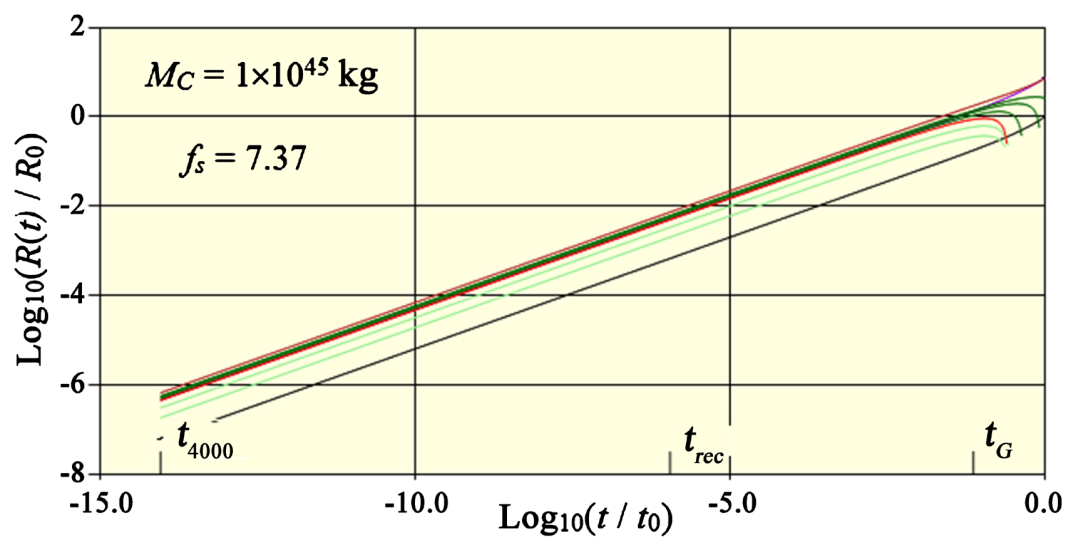

Figure 4. Solution with $t_{s}=4000 \mathrm{~s}, M_{C}=1 \times 10^{45} \mathrm{~kg}$, and $f_{s}=7.37$.

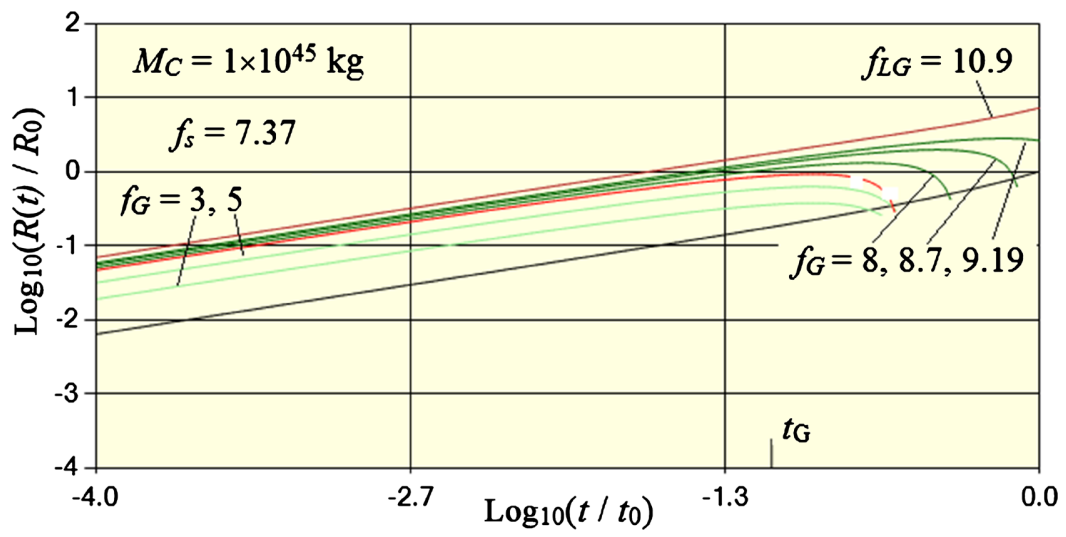

Figure 5. Detail of the solution with $t_{s}=4000 \mathrm{~s}, M_{C}=1 \times 10^{45} \mathrm{~kg}$, and $f_{s}=7.37$.

cluster gas came into existence during nucleosynthesis already at that density.

This same result is shown in more detail in Figure 5.

In this case, we have included 2 internal galaxies in addition to a set of 3 external galaxies.

We have also included a curve representing the local group (LG) which turns out to be an interesting problem. The present-day value of $f_{L G}\left(t_{0}\right)=7.5$ and by adjusting the parameter, we obtain a starting value of $f_{s}=10.9$. The curves are shown in Figure 6.

As can be seen, the prediction is that the LG is still receding. The predicted velocity is $866 \mathrm{~km} \cdot \mathrm{s}^{-1}$ away from the cluster whereas the observed velocity is in the range $100-400 \mathrm{~km} \cdot \mathrm{s}^{-1}$ towards the cluster.

Its velocity away from the cluster due to just the scaling would be about 1100 $\mathrm{km} \cdot \mathrm{s}^{-1}$ so the local group is experiencing the gravitation tug of the cluster as can also be seen by comparing the two curves in the figure. The tug, however, is not enough to drag it in. If we ask now ask what value of $f_{s}$ would result in a value of $-200 \mathrm{~km} \cdot \mathrm{s}^{-1}$, we obtain $f_{G}=9.19$ which is not a great deal smaller than 10.9 . The corresponding present-day distance, however, is about $36 \%$ of the actual LG distance which is quite a bit different. A first sight, it appears that the model does not correctly predict the motion of the LG but we do not believe that to be 


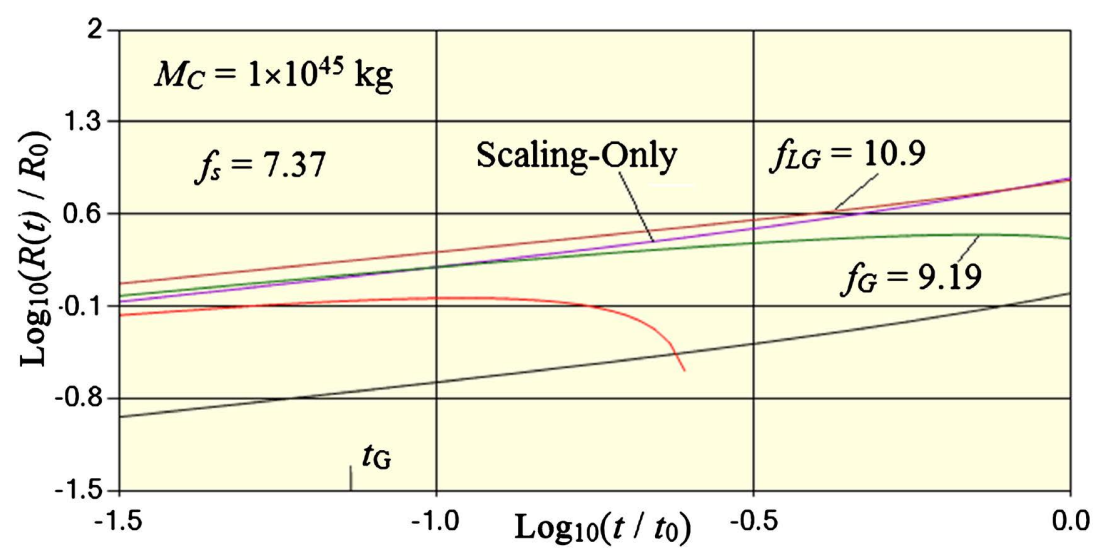

Figure 6. Model solution for the local group.

the case. We tried a considerable number of parameter combinations and found that it is impossible to obtain a solution with the LG at its present-day position with its present-day velocity of approach. Either the LG escapes or is pulled in much sooner. The conclusion we come to is that at some point during its history, the LG received a boost in the direction of the cluster because it is just too far away to overcome the expansion of the universe otherwise.

Another prediction we can check is the velocity of the galaxies at the time they cross the boundary of the cluster assuming that it remains constant in size after the zero-velocity point. For the galaxies with $f_{G}=8$ and 8.7 , the results are $-229 \mathrm{~km} \cdot \mathrm{s}^{-1}$ and $-660 \mathrm{~km} \cdot \mathrm{s}^{-1}$ respectively. These are quite reasonable predictions as we will see shortly. At present, the average cluster is about $4 \%$ the size of an average supercluster, [1]. At the time $t=4000 \mathrm{~s}$, with the initial compaction of 7.37 , an average cluster would have been about $30 \%$ the size of an average supercluster which again is not unreasonable.

What we have shown is, first that the cluster could not have come into existence via an accretion process beginning at $t=t_{G}$ (or later) and that a starting time even as early as $t=4000 \mathrm{~s}$ requires an initial compaction ratio of about 1.35 or an initial density of $2.5 \mathrm{~m}^{-3}$ in present-day terms. As we saw, this initial compaction results in the cluster reaching its ZVP at about $t=t_{G}$ and also allows for a range of external galaxy capture out to a distance on the order of 8.7 times the radius of the cluster.

Throughout our development of the new model, causality was always a major consideration so it is important to consider the implications here. Even though at present all the points in the cluster can exchange signals, that was very definitely not the case at the time of nucleosynthesis. In Figure 7, we show two curves that illustrate the issue.

The straight gray line is the general causality limit fixed by the age of the universe. As just noted, at the time of nucleosynthesis the cluster was far larger than this limit. As argued earlier, its existence is the result of a non-causal imprint on the vacuum that regulated nucleosynthesis rather than the result of causal processes. The curved gray line is the limiting distance beyond which a 


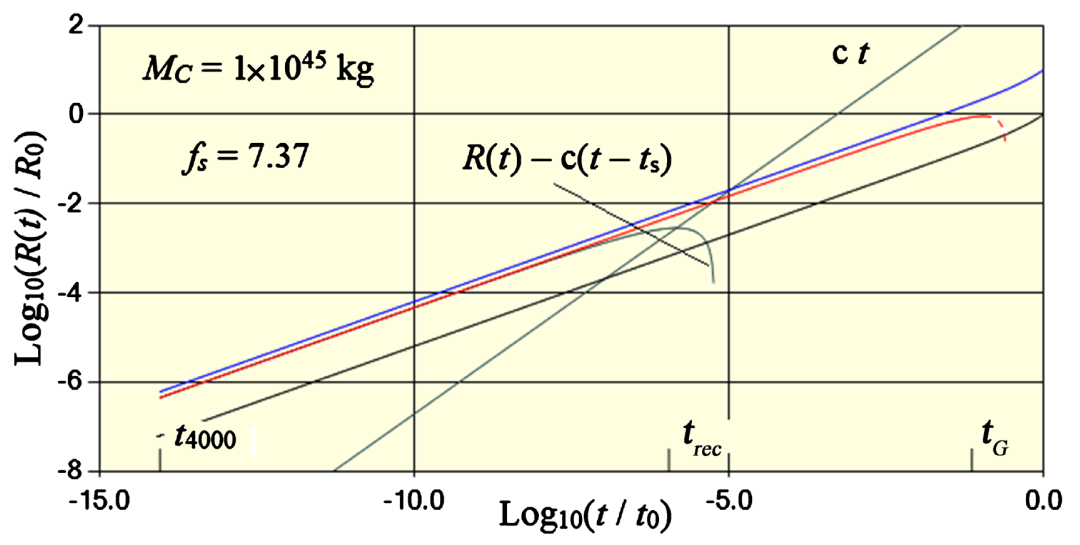

Figure 7. Causality constraints.

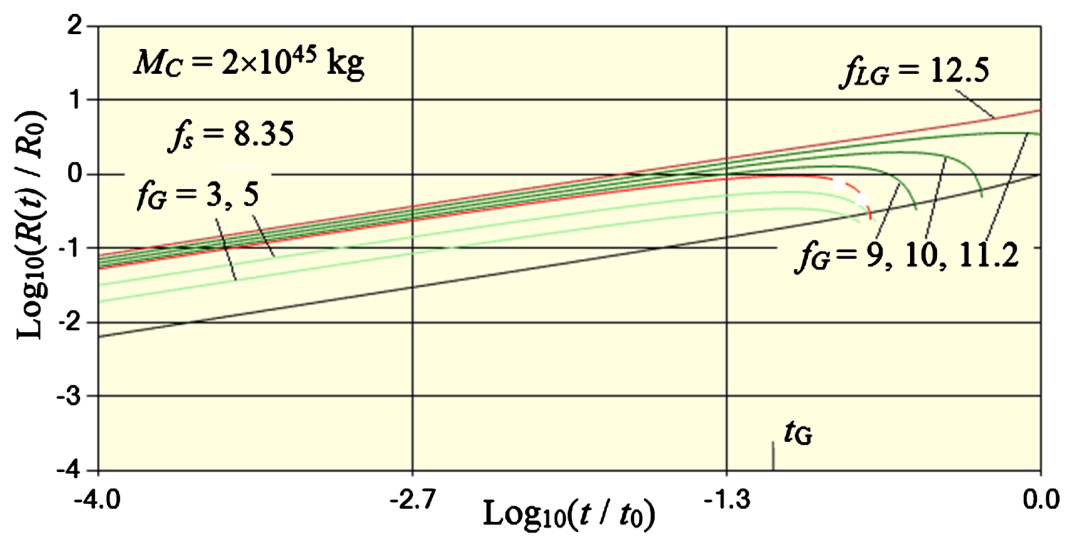

Figure 8. Detail of the solution with $t_{s}=4000 \mathrm{~s}, M_{C}=2 \times 10^{45} \mathrm{~kg}$, and $f_{s}=8.35$.

point of the surface will have no awareness. This means, for example, that the points on the surface initially had no awareness of the mass of the cluster. They would, however, be aware of an immediate gradient in the density at the surface. What is apparent from the figure, however, is that the motion of the surface was controlled entirely by the velocity of expansion of the universe until a time somewhat later than $t / t_{0}=10^{-5}$ so the fact that the surface points were unaware of the total mass of the cluster before that time was of no consequence. In fact, from Figure 5 we see that the surface didn't really begin to respond to the mass of the cluster until $t / t_{0} \geq 10^{-3}$, and by then, the whole of the cluster was well inside the causality limit.

Referring back to the discussion of Section 4, the organization necessary to initiate rotation via standard processes could not have even begun to exist until sometime well after $t / t_{0}=10^{-5}$. The conclusion is the same as before. If a cluster is showing rotation, the rotation is primordial and it is the vacuum that is rotating carrying the cluster matter along with it.

At this point for comparison, we now show in Figure 8 the results for a mass of $M_{C}=2 \times 10^{45} \mathrm{~kg}$.

Comparing with Figure 5, we see that, although the parameters are different, the curves are essentially the same so, at this point, there is no justification for 
preferring any one total mass over any other.

An interesting point about these results is that the cluster reaches zero radial velocity and hence its present-day size at a time $t=t_{G}$ independent of the cluster mass and this seems to be a general result. For early times, the system in question expands as a non-interacting gas. For later times, detailed gravitational processes become dominant.

Earlier we stated that vacuum energy can account for the phenomena attributed to dark matter. In this case, the issue is the deficit mass problem if there is one. The model calculations are dependent only on the total mass. If we assume that the present-day density of particles is correct, then the deficit amounts to 4 $\times 10^{44} \mathrm{~kg}$ in the one case and $1.8 \times 10^{45} \mathrm{~kg}$ in the other. We now consider the vacuum energy density. Its present-day value is $2 \times 10^{-10} \mathrm{j} \cdot \mathrm{m}^{-3}$ which is equivalent to a mass density of $2.2 \times 10^{-27} \mathrm{~kg} \cdot \mathrm{m}^{-3}$. The details of how the vacuum imprint regulates the creation of matter are not known at present but if the vacuum density reflects the created particle compaction, then, in the lower mass case, the vacuum density would have been larger than the background by a factor of $(10 / 7.37)^{3}=2.5$. If the vacuum density continued to increase during the run-up to $t_{G}$, the density increase would be by a factor of $10^{3}$. Given the accepted particle density, the density of the deficit was $3 \times 10^{-25} \mathrm{~kg} \cdot \mathrm{m}^{-3}$ which we compare with a vacuum density somewhere in the range of $5.5 \times 10^{-27}-2.2 \times 10^{-24} \mathrm{~kg} \cdot \mathrm{m}^{-3}$. In the lower mass case, the vacuum density ratio would be 139 and in the higher mass case, it would be 485 so the vacuum can easily account for the deficit.

\section{Cluster Equilibrium}

We will now consider the problem of the stability of the cluster ICM after the ZVP. Actually, calling it the intracluster gas, as is commonly the case, is a misnomer. The proton gas is not something that is inside the cluster, the proton gas is the cluster.

One of the major mysteries of galaxy clusters is how to account for the observed temperature of the gas which is variously reported, see e.g. [5], to be in the range of $10<T<100 \mathrm{MK}$. Up until the time of recombination, the ionized gas would have been in thermal equilibrium with the CMB so, at $t_{r e c}$, the temperature would have been on the order of $T=4000 \mathrm{~K}$ and since the temperature decreases as a result of the expansion, the present-day energy of the gas cannot be primordial. Further, before $t_{G}$ there were no sources so the gas energy must be a consequence of sources that came into existence after passing the ZVP in the evolution of the cluster.

To shed some light on this and related problems, we will consider an equilibrium model for the cluster. Consider a volume of the ICM gas located at a distance $r$ from the center of the cluster. The force acting on that volume due to the mass of the cluster would be

$$
F=\frac{G m(r) \rho(r)}{r^{2}}
$$


where $m(r)$ is the fraction of the total mass inside the sphere at radius, $r$. In the absence of any opposing forces, during a time interval $\Delta t$, the volume would move a distance $\Delta r$ towards the center so an amount of work per unit time would be done equal to

$$
W=\frac{G m(r) \rho(r)}{r^{2}} \frac{\Delta r}{\Delta t} .
$$

To get a characteristic velocity, we consider the potential acting on the volume which is given by

$$
U=\frac{G m(r) \rho(r)}{r}
$$

so a volume dropped in from infinity would thus have a velocity equal to

$$
\frac{\Delta r}{\Delta t}=\sqrt{\frac{G m(r)}{r}}
$$

To achieve equilibrium, this work must be balanced by a pressure gradient in the gas and equilibrium requires that the work done must be balanced by incoming radiation,

$$
\frac{G m(r) \rho(r)}{r^{2}} \sqrt{\frac{G m(r)}{r}}=\rho(r) \varepsilon(r)
$$

where $\varepsilon(r)$ is an external energy source with the units of $\mathrm{j} \cdot \mathrm{s}^{-1} \cdot \mathrm{kg}^{-1}$. Solving for $m(r)$, we have

$$
m(r)=\frac{\varepsilon(r)^{2 / 3}}{G} r^{5 / 3}
$$

We will now make the assumption that $\varepsilon(r)=\varepsilon_{0}$ is a constant (note that the units are per $\mathrm{kg}$ rather than per volume) so we have

$$
\frac{\mathrm{d} m(r)}{\mathrm{d} r}=\frac{5}{3 G} \varepsilon_{0}^{2 / 3} r^{2 / 3}=4 \pi r^{2} \rho(r) .
$$

The second equality follows from the definition of $m(r)$. Solving for the density gives

$$
\rho(r)=\frac{5}{12 \pi G} \frac{\varepsilon_{0}^{2 / 3}}{R_{0}^{4 / 3}}\left(\frac{R_{0}}{r}\right)^{4 / 3} \equiv \bar{\rho}\left(\frac{R_{0}}{r}\right)^{4 / 3}
$$

The total mass is

$$
M_{C}=\int_{0}^{R_{0}} \mathrm{~d} r 4 \pi r^{2} \rho(r)
$$

and after substituting, we find

$$
\varepsilon_{0}=\frac{\left(G M_{C}\right)^{3 / 2}}{R_{0}^{5 / 2}} .
$$

The acceleration of the volume must be balanced by the pressure gradient so we have 


$$
\frac{1}{\rho(r)} \frac{\mathrm{d} p(r)}{\mathrm{d} r}=-\frac{G m(r)}{r^{2}}
$$

which yields

$$
\frac{\mathrm{d} p(r)}{\mathrm{d} r}=\frac{-5}{12 \pi G} \frac{\varepsilon_{0}^{4 / 3}}{R_{0}^{5 / 3}}\left(\frac{R_{0}}{r}\right)^{5 / 3} .
$$

Solving for pressure gives

$$
p(r)=\frac{5}{8 \pi G} \frac{\varepsilon_{0}^{4 / 3}}{R_{0}^{2 / 3}}\left(\frac{R_{0}}{r}\right)^{2 / 3} \equiv \bar{p}\left(\frac{R_{0}}{r}\right)^{2 / 3} .
$$

At this point, we have everything needed to understand the equilibrium but we would also like to know the temperature which we can determine if we know the equation of state of the gas. Not knowing the actual equation of state, we will assume an ideal gas. The ideal gas law states that $p / \rho=k_{B} T /\left(\mu m_{p}\right)$ where $\mu=1 / 2$ for fully ionized hydrogen. We have then

$$
T(r)=\frac{p(r)}{\rho(r)} \frac{\mu m_{p}}{k_{B}}=6.05 \times 10^{-5} \frac{3}{2}\left(\varepsilon_{0} R_{0}\right)^{2 / 3}\left(\frac{r}{R_{0}}\right)^{2 / 3} \equiv \bar{T}\left(\frac{r}{R_{0}}\right)^{2 / 3} .
$$

Finally, we want to solve for the velocity of a galaxy that falls into the cluster. By the time of $t=t_{G}$, the scaling contribution to the acceleration was negligible and can be dropped. On the other hand, we do need to consider the pressure gradient and the drag force. The equation of motion is

$$
\frac{\mathrm{d}^{2} r_{G}}{\mathrm{~d} t^{2}}=-\frac{G m(r)}{r^{2}}+a_{p}(r)+a_{D}(r)
$$

The force exerted by the pressure gradient is

$$
F_{p}=\frac{\mathrm{d} p}{\mathrm{~d} r} \Delta r A=\frac{5}{12 \pi} \frac{G M_{C}}{R_{0}^{5}} \Delta r A\left(\frac{R_{0}}{r}\right)^{5 / 3} .
$$

Using the MW as an example,

$$
\Delta r A=O\left(R_{M W}^{3} / 2\right)
$$

so we have

$$
a_{p}(r)=\frac{F_{p}(r)}{M_{M W}} \leq 2.6 \times 10^{-15}\left(\frac{R_{0}}{r}\right)^{5 / 3} \mathrm{~m} \cdot \mathrm{s}^{-2} .
$$

This result is, however, an overestimate because the formula used assumes that the object is a solid body which it clearly is not. For comparison, $G M_{C} / R_{0}^{2}=1.5 \times 10^{-11} \mathrm{~m} \cdot \mathrm{s}^{-2}$ so we find that the acceleration due to the pressure gradient is negligible except possibly at the center of the cluster.

The drag force is

$$
F_{D}=\frac{1}{2} C_{D} \rho A v^{2} .
$$

Assuming a drag coefficient no greater than 0.1 which is appropriate for a solid body with a very large Reynold's number, and assuming a velocity of 700 
$\mathrm{km} \cdot \mathrm{s}^{-1}$ we find a drag force no more than 4 times that pressure gradient force so it too can be neglected. The equation of motion of the galaxy is then

$$
\frac{\mathrm{d}^{2} r_{G}}{\mathrm{~d} t^{2}}=-\frac{G m(r)}{r^{2}}=-\varepsilon_{0}^{2 / 3} \frac{1}{r^{1 / 3}} .
$$

We now just plug in the numbers and calculate the results. The curves are shown in Figure 9 and the magnitudes are listed in Table 1. The normalized curves for the density, pressure, and temperature are independent of the assumed mass. The velocity curve does vary slightly with the mass but not enough to warrant including multiple curves.

These results are in general agreement with observation, [4]. The density, and the pressure, rise towards the center with a strong peak, and the temperature drops towards the center. The observed temperatures lie in the range $10<T<100 \mathrm{MK}$ so for a total mass of $1 \times 10^{45}$, the predicted value is in agreement. The corresponding energy, $\frac{3}{2} k_{B} T$, is on the order of $12 \mathrm{KeV}$. The proton gas has an extremely low density, fairly high energy, and it is charged and its equation of state could differ from that of an ideal gas.

At this point, we can now see that with a total mass of $2 \times 10^{45} \mathrm{~kg}$, the predicted temperature is almost a factor of 2 too large and an even bigger problem is the magnitude of the energy flux needed to establish equilibrium. These together support the idea that the total mass is significantly less than the accepted value.

Before getting to the energy problem, we will finish with the galaxy velocity predictions. The original motivation for dark matter was to explain what was thought to be a deficit in the total mass needed to explain the observed velocities

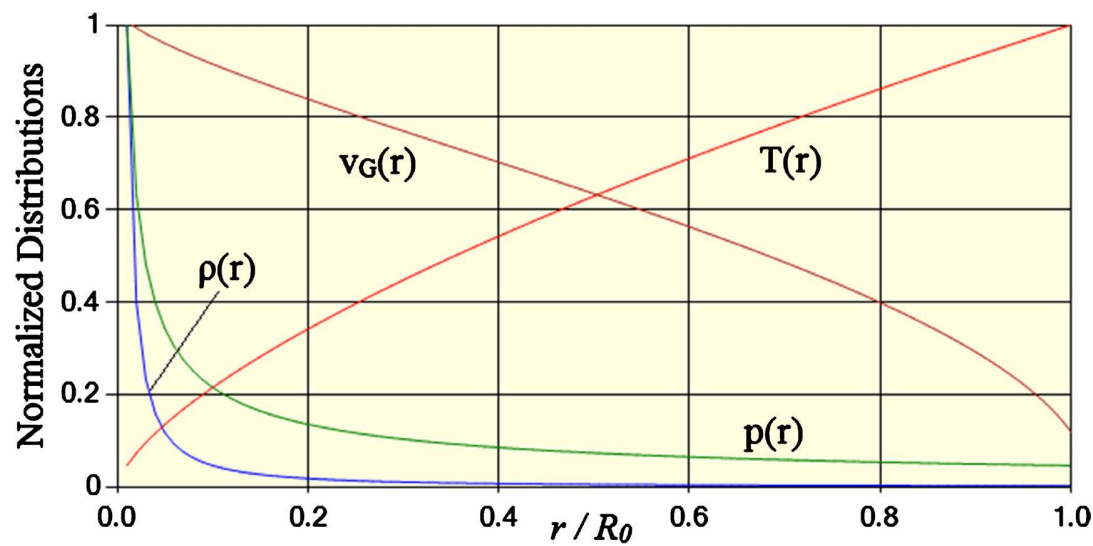

Figure 9. Calculated model curves normalized to unit magnitude.

Table 1. Equilibrium solution parameter values.

\begin{tabular}{cccccc}
\hline$M_{C}(\mathrm{~kg})$ & $\varepsilon_{0}\left(\mathrm{j} \cdot \mathrm{s}^{-1} \cdot \mathrm{kg}^{-1}\right)$ & $\bar{\rho}\left(\mathrm{kg} \cdot \mathrm{m}^{-3}\right)$ & $\bar{p}\left(\mathrm{n} \cdot \mathrm{m}^{-2}\right)$ & $\bar{T}\left(10^{6} \mathrm{~K}\right)$ & $\langle T\rangle\left(10^{6} \mathrm{~K}\right)$ \\
\hline $1 \times 10^{45}$ & $1.44 \times 10^{-5}$ & $4.23 \times 10^{-25}$ & $6.25 \times 10^{-13}$ & 89 & 64 \\
$2 \times 10^{45}$ & $4.08 \times 10^{-5}$ & $8.47 \times 10^{-25}$ & $2.51 \times 10^{-12}$ & 178 & 127
\end{tabular}


of the cluster galaxies. In Figure 10, we show numerical solutions of (7-20).

The data will be explained in detail in the next section. The red and blue dots indicate the observed radial velocities of the member galaxies of the Virgo cluster. The center value, $1100 \mathrm{~km} \cdot \mathrm{s}^{-1}$, is the velocity of recession of the cluster from our location. The black lines are the predictions. The upper pairs are the radial velocity of a galaxy traveling away from us along our line of sight towards the center of the cluster starting at the cluster surface closest to us. The lower pair are the velocities for a galaxy again traveling along our line of sight but this time, towards us having started at the far surface of the cluster.

What we find is that the velocities increase with total mass and that the smaller total mass prediction does account for the observed maximum velocities. We also see that the velocities predicted based on the larger total mass are much larger than the observed velocities which is the best indication we have that the current dark matter estimate of the total mass is too large. Finally, although we don't show it, the velocities predicted based on the accepted particle mass alone $\left(6 \times 10^{44} \mathrm{~kg}\right)$ are marginally too low. We also note that velocities close to the center could be damped if the increasing pressure gradient and drag forces become significant.

We will now return to the energy flux problem. We saw that in the lower mass case, the necessary energy flux density is $\varepsilon_{0}=1.44 \times 10^{-5} \mathrm{j} \cdot \mathrm{s}^{-1} \cdot \mathrm{kg}^{-1}$. Multiplying by the total mass of the cluster, this becomes $E_{0}=4.54 \times 10^{47} \mathrm{j} \cdot \mathrm{yr}^{-1}$ which is equivalent to the complete conversion of 2.5 Suns per year into pure energy. The only sources with energy outputs remotely in that range are supernovae with outputs as much as $10^{44} \mathrm{j}$ and supermassive black holes. Powering the equilibrium

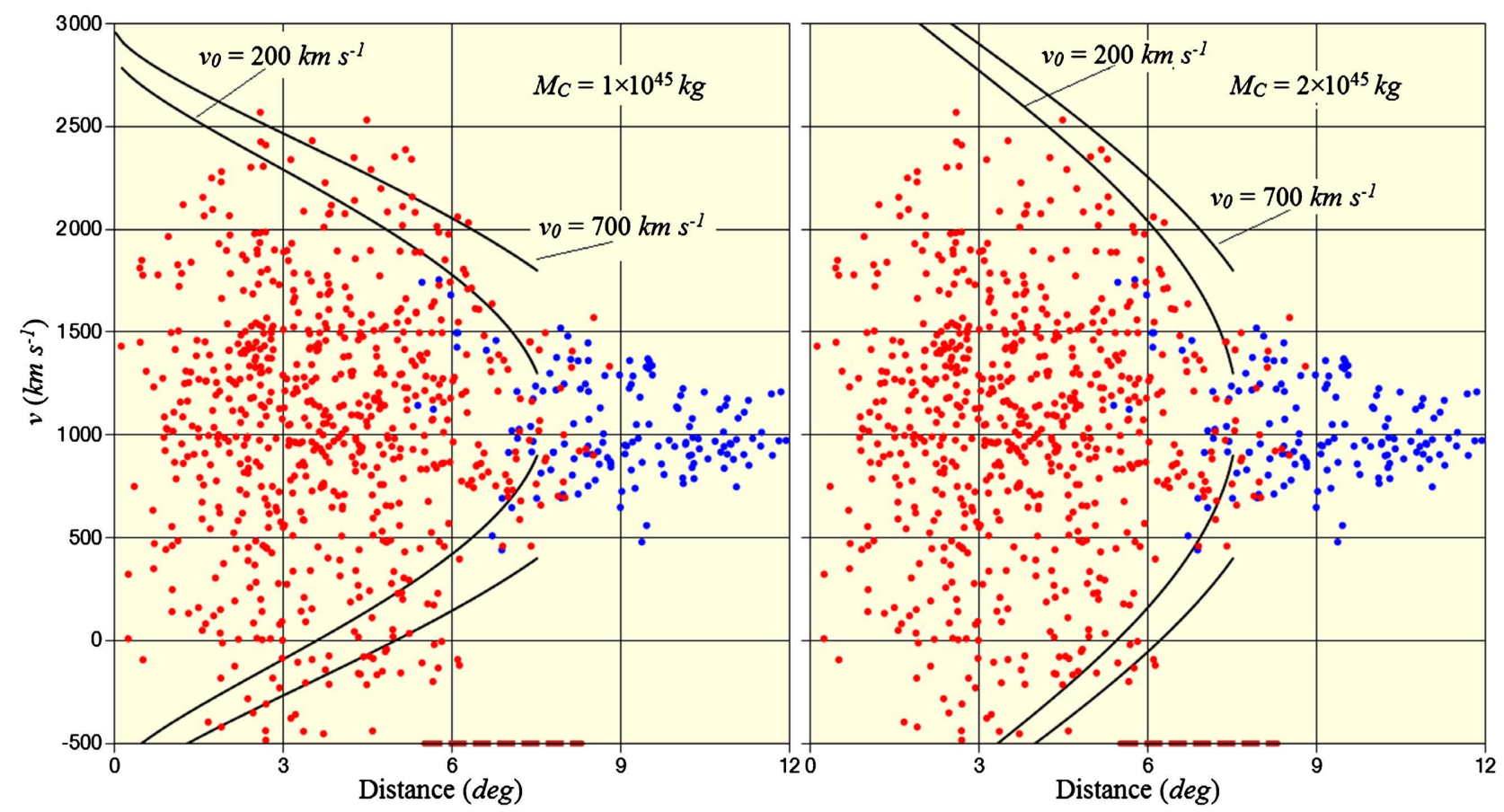

Figure 10. Galaxy velocity predictions compared with the observed radial velocity distribution. 
with supernovae would require the completer absorption of the total output of 4540 supernovae per year. Supermassive black holes produce more output but they are much rarer. All in all, it doesn't seem possible to account for the necessary energy and surely, if there was such a high flux, it would be obvious to observers.

It would appear that powering the equilibrium is a real problem but now we come to the crux; equilibrium is, in fact, impossible and without equilibrium, our picture of the cluster evolution changes considerably.

Referring to (7 - 12), we see that a small increase in the energy flux results in an increase in the magnitude of the pressure gradient which, in turn, results in an outward acceleration of the gas particles so $R_{0}$ would get larger. But according to $(7-10)$, the energy flux needed for equilibrium varies inversely with $R_{0}$ so we find that the equilibrium is unstable. For a given mass and size, equilibrium can only exist for a single precise value of the flux, and any small variance in that flux results in an acceleration away from equilibrium. The cluster, of course, has no control over the flux so a state of general equilibrium is impossible.

This fact now changes our original idea of the post-zero-velocity evolution of the cluster. Clusters do not undergo virialization after the zero-velocity point because equilibrium is impossible. Instead, we suppose that shortly after the ZVP, the cluster underwent further expansion as a result of the extreme output of radiation during the early phase of galaxy and star formation. Later, the cluster would have begun to shrink as the energy flux leveled off and begin to decline with the cluster size being determined by both the energy flux that remained and the compressive heating of the gas.

The final stage of this compaction is an adiabatic equilibrium in the absence of radiation. The equations are given by (7-11), $\mathrm{d} m(r) / \mathrm{d} r=4 \pi \rho(r) r^{2}$, and $p(r) / \rho(r)^{\gamma}=A=$ const where $\gamma=5 / 3$. We introduce dimensionless coordinates and parameters,

$$
\begin{gathered}
\xi=r / R_{0} \\
m(\xi)=M_{C} \bar{m}(\xi) \\
p(\xi)=\frac{G M_{C}^{2}}{R_{0}^{4}} \bar{p}(\xi) \\
\bar{A}=\left(\frac{G R_{0} M_{c}^{1 / 3}}{A}\right)^{3 / 5} .
\end{gathered}
$$

The equations then become,

$$
\begin{gathered}
\frac{\mathrm{d} \bar{p}(\xi)}{\mathrm{d} \xi}=-\bar{A} \frac{\bar{m}(\xi)}{\xi^{2}} \bar{p}(\xi)^{3 / 5} \\
\frac{\mathrm{d} \bar{m}(\xi)}{\mathrm{d} \xi}=4 \pi \bar{A} \xi^{2} \bar{p}(\xi)^{3 / 5}
\end{gathered}
$$




$$
\bar{A}=\left(4 \pi \int_{0}^{1} \mathrm{~d} \xi^{\prime} \xi^{\prime 2} \bar{p}\left(\xi^{\prime}\right)^{3 / 5}\right)^{-1} .
$$

We now make one further simplification. We define 2 constant scaling factors by $\bar{p}(\xi)=p_{s}(\xi) s_{1}$ and $\bar{m}(\xi)=m_{s}(\xi) s_{2}$ and then chose values of the constants to eliminate the $\bar{A}$ dependence in the equations. The result is $s_{2}=1 / \bar{A}^{5}$ and $s_{1}=s_{2}^{2}$. Finally, we define $A_{s}=\bar{A}^{5}$ and end up with

$$
\begin{aligned}
& \frac{\mathrm{d} p_{s}(\xi)}{\mathrm{d} \xi}=-\frac{m_{s}(\xi)}{\xi^{2}} p_{s}(\xi)^{3 / 5} \\
& \frac{\mathrm{d} m_{s}(\xi)}{\mathrm{d} \xi}=4 \pi \xi^{2} p_{s}(\xi)^{3 / 5} \\
& A_{s}=4 \pi \int_{0}^{1} \mathrm{~d} \xi^{\prime} \xi^{\prime 2} p_{s}\left(\xi^{\prime}\right)^{3 / 5}
\end{aligned}
$$

with

$$
\begin{gathered}
p(\xi)=\frac{G M_{C}^{2}}{R_{0}^{4}}\left(A_{s}^{-2} p_{s}(\xi)\right) \\
\rho(\xi)=\frac{M_{C}}{R_{0}^{3}}\left(A_{s}^{-1} p_{s}^{3 / 5}\right) \\
T(\xi)=\frac{\mu m_{p}}{k_{B}} \frac{G M_{C}}{R_{0}}\left(A_{s}^{-1} p_{s}(\xi)^{2 / 5}\right) .
\end{gathered}
$$

We solve the equations numerically with the results shown in Figure 11.

In this case, there are no sources and the equilibrium is stable. Comparing with Figure 9, we see that the curves are quite different. Particularly noticeable is that the temperature is increasing towards the center instead of decreasing. The value of $A_{s}=0.348$. At the outer edge, (7-24) give values of

$p(1)=2.6 \times 10^{-13} \mathrm{n} \cdot \mathrm{m}^{-2}, \quad \rho(1)=5.8 \times 10^{-25} \mathrm{~kg} \cdot \mathrm{m}^{-3}, \quad T(1)=27 \times 10^{6} \mathrm{~K}, \quad$ and comparing with Table 1 , we see that the magnitudes of the pressure and density are similar but the adiabatic temperature is about a factor of 3 lower.

Today, observations reveal curves that are more in line with those of Figure 9 from which we conclude that the cluster is still shrinking and will continue to do

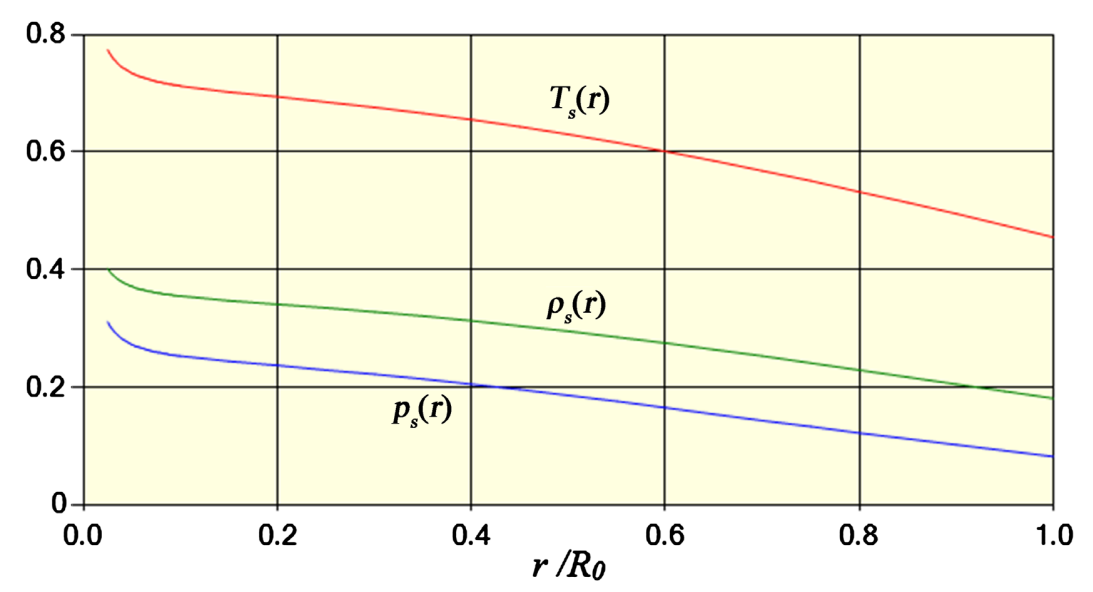

Figure 11. Adiabatic equilibrium solution. 
so until the cluster reaches adiabatic equilibrium. In the literature, the Virgo cluster is often referred to as being young but that isn't the case, all clusters have the same age.

To reiterate, the present-day energy of the ICM is the result of the intense radiation consequent to star and black hole formation during the early stages of galaxy evolution. A numerical study of this evolution is beyond the scope of this paper and will be addressed in a future paper.

\section{Distribution of Cluster Galaxies}

Having dealt with the accretion and stability, we will now finish our discussion of clusters with an examination of the actual distribution of the galaxies making up the membership of the Virgo cluster. The basis of our study is the Extended Virgo Cluster Catalogue (EVCC), [6] which is an extension of the original VCC [7]. This extended catalogue covers a section of the sky 5.2 times larger than that of the VCC which is important in this case because it allows us to separate the internal and external populations. One prediction of our model is that there are two distinct populations of galaxies within the cluster and in this section, we will review evidence that supports this idea. The authors of [8] also found evidence that supports the same idea.

The terms ETG (early-type galaxy) and LTG (late-type) have long been in use and are used in the EVCC catalogue. In the new model, all the galaxies originated during nucleosynthesis so the temporal distinction between the two does not exist but we will continue to use the terms as a convenient shorthand for elliptical and lenticulars on the one hand and spirals on the other.

In the EVCC, the authors separate the counted galaxies into those that were common to both the EVCC and the VCC and those that were not in the VCC. The problem with this from the point of view of our study is that many galaxies were observed to be inside the cluster boundary by the EVCC group but were not recorded in the VCC catalogue. Our first step, then, was to separate all the galaxies into two new categories consisting of all those inside the cluster boundary and all those outside the boundary.

A second categorization used by the EVCC group separates the galaxies into those that are definitely members of the cluster and those that are only possibly members. The following Table 2 summarizes the counts.

We have shown the breakdown of the ETGs for later reference. Clearly, the dE and dEN dominate the counts. We haven't shown a similar breakdown for the LTGs because there is no dominant type and the distributions are all similar. In Figure 12, we show the entire collection.

In what follows, we will need to know the position of the center of the cluster. We first estimated its position by finding an approximation of the geometric center of the cluster boundary shown in Figure 12. Afterward, we calculated the average position of all the galaxies inside the boundary and obtained essentially the same result. The position found does not, however, coincide with the point 
Table 2. Summary of galaxy type counts included in the EVCC.

\begin{tabular}{cccccc}
\hline Morphology & Mem Inside & Mem Outside & Possible Inside & Possible Outside & Totals \\
\hline E & 28 & 1 & 7 & 2 & 38 \\
dE & 127 & 23 & 44 & 10 & 204 \\
dEN & 234 & 30 & 21 & 5 & 290 \\
S0 & 39 & 4 & 13 & 8 & 64 \\
SB0 & 12 & 2 & 3 & 0 & 17 \\
dS0 & 41 & 15 & 12 & 11 & 79 \\
dSN & 32 & 4 & 4 & 1 & 41 \\
ETGs & 513 & 79 & 104 & 37 & 733 \\
LTGs & 150 & 62 & 61 & 109 & 382 \\
Irregulars & 120 & 66 & 64 & 142 & 392 \\
Edge-on & 27 & 11 & 11 & 33 & 82 \\
Totals & 810 & 218 & 240 & 321 & 1589 \\
\hline
\end{tabular}

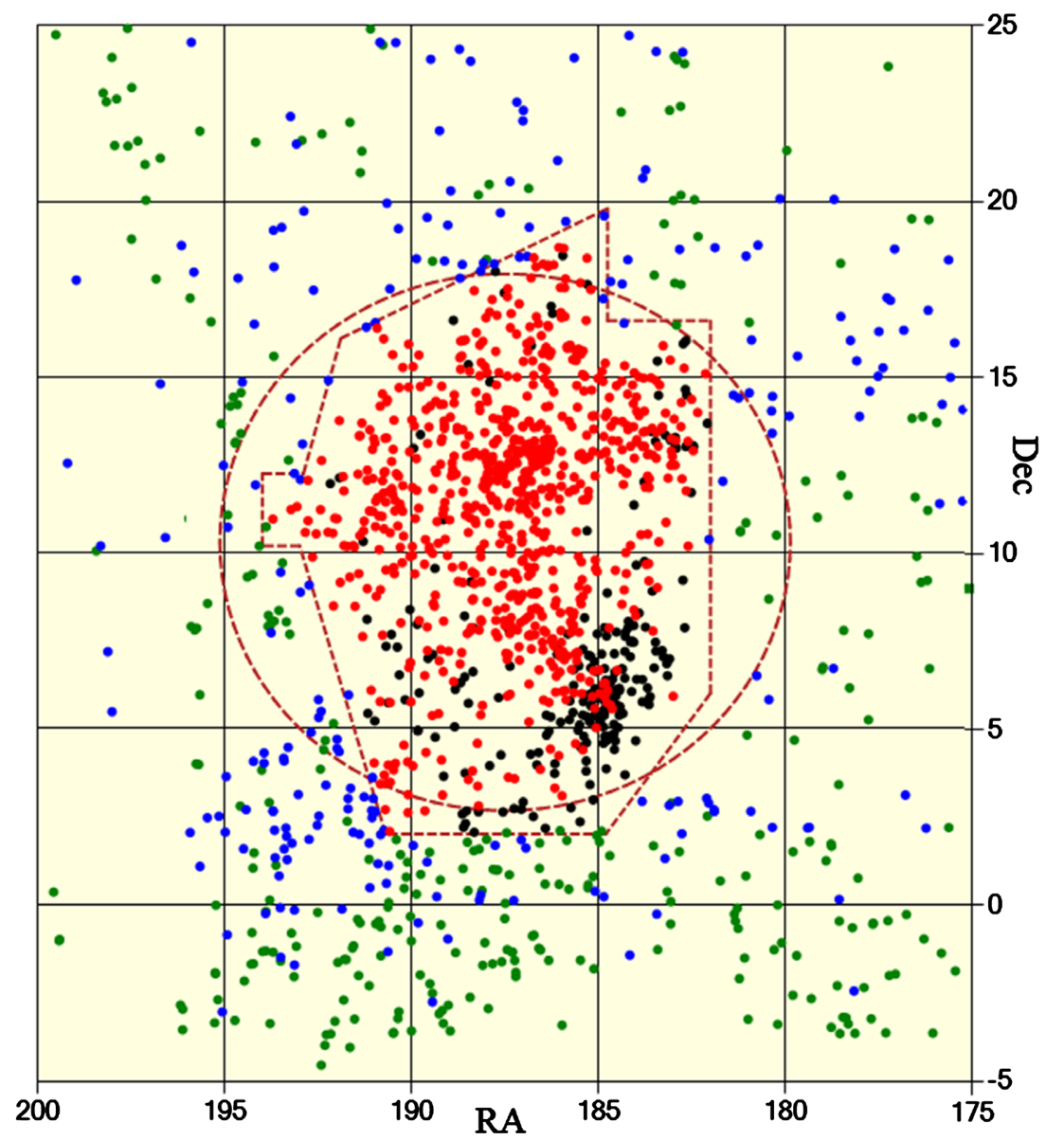

Figure 12. The entire EVCC catalogue. The red dashed polygon is an approximation to the VCC boundary. The red dashed circle has a radius $2.2 \mathrm{Mpc}$ which is the accepted radius of the cluster. The red and blue dots indicate member galaxies inside and outside the boundary respectively. Black and green are the corresponding colors for the possibles. 
of the highest density of the galaxies which may or may not be of any significance.

For our study, we are primarily interested in the members as opposed to the possibles. Next, because we are interested in the distributions by type, we eliminated the edge-ons from consideration. There are not many of these and they are pretty evenly distributed in any case. Finally, from the table, we see that the irregulars have a distribution very similar to the LTGs so we generally lumped these two groups together.

In Figure 13, the ETGs are shown to the left and the LTGs plus irregulars on the right.

From this figure, we see the concentration of the ETGs inside the cluster boundary with the $\mathrm{dE}$ and $\mathrm{dENs}$ making up the bulk of the total. Outside the boundary, the density of ETG's is low compared to that of the LTGs and tends to concentrate close to the cluster. The LTGs, on the other hand, are much more evenly distributed across the entire study area so in both cases the basic distribution pattern tends to flow smoothly across the cluster boundary.

For the next few figures, we divided the study area into a series of concentric rings of equal width. The histograms show the various counts.

In Figure 14, we compare the distributions of the ETGs and the LTGs.

Outside the boundary, the distributions are similar with a few more LTGs than ETGs. Inside, the ETGs are dominant. The catalogue presents a projection of the galaxies onto a plane perpendicular to the line of sight and it would be useful to have some idea of how this projected image reflects the 3-dimensional distribution of galaxies. To find out, we assumed a uniform density of galaxies
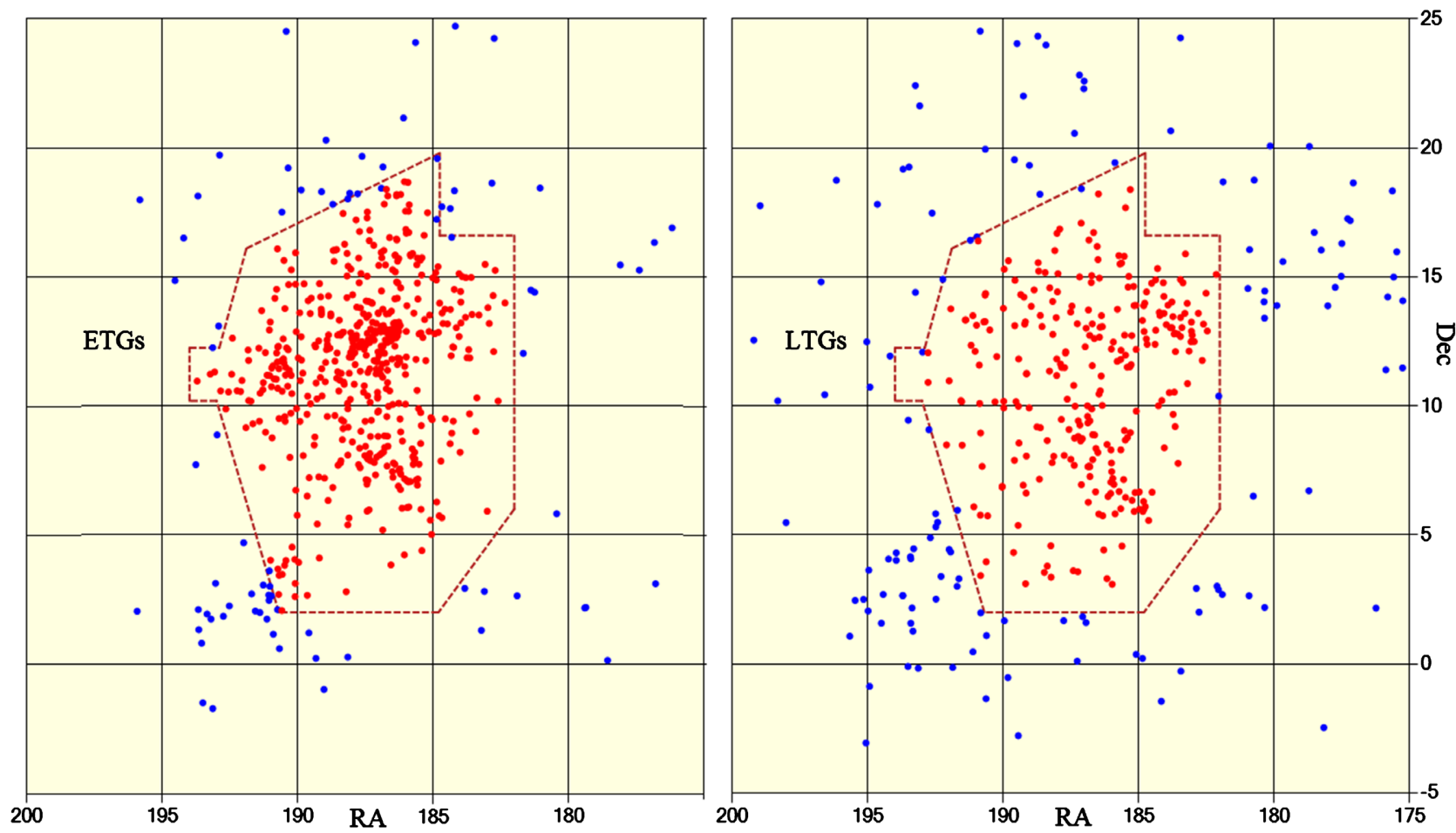

Figure 13. Distribution of member galaxies, ETGs on the left, LTGs plus irregulars on the right. 


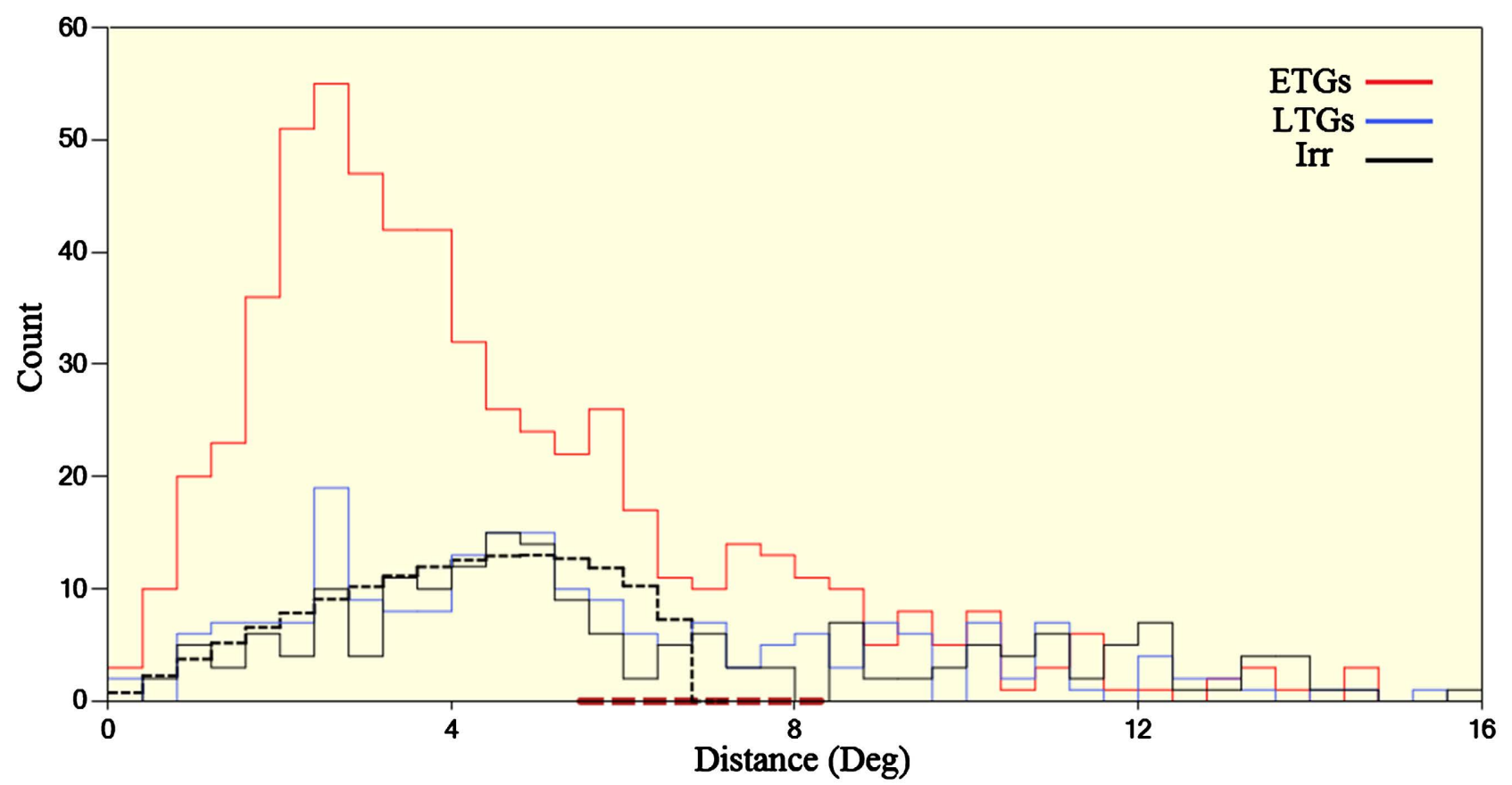

Figure 14. Distribution of ETGs, LTGs, and irregulars with a bin count of 40 . The dashed red line at the bottom of the chart indicates the limits of the cluster boundary. The dashed black line is described in the text.

and then projected that distribution onto the perpendicular surface with an arbitrary normalization. The result is the dashed black line. In this case, the normalization was adjusted to roughly match the LTG curve. It is the shape of the curve that is significant and what this tells us is that the LTG density is nearly uniform inside the boundary which is what one would expect for a population of initially external galaxies that underwent capture by the cluster over a long period.

The shape of the ETG curve, on the other hand, is not even remotely similar to that of the uniform density curve which is an indication that the ETGs and LTGs form two distinct populations with the bulk of the ETGs having come into existence inside the cluster beginning at the time of nucleosynthesis $\left(f_{G}<7.37\right.$ in Figure 5.)

In Figure 15, we show the distribution of the various types of ETGs.

We see that $\mathrm{dE}$ and dEN are the dominant types. To show this more clearly, in Figure 16 we have combined the $\mathrm{dE}$ and dEN types into one group and the remaining ETGs into another.

This clearly shows that the $\mathrm{dE}$ and $\mathrm{dEN}$ are the dominant types. It also appears at first sight that the distributions are different but that is solely a consequence of the relative counts. In Figure 17, we show the same groupings but with the E, etc. grouping scaled in magnitude by the ratio of the total numbers of galaxies in each group. From this, we see that the distributions are essentially the same which implies that we can realistically treat the ETGs as a single group as far as their origin is concerned.

In Figure 18, we show the distribution of LTGs by type.

The collective distribution is not dominated by any particular type which is 


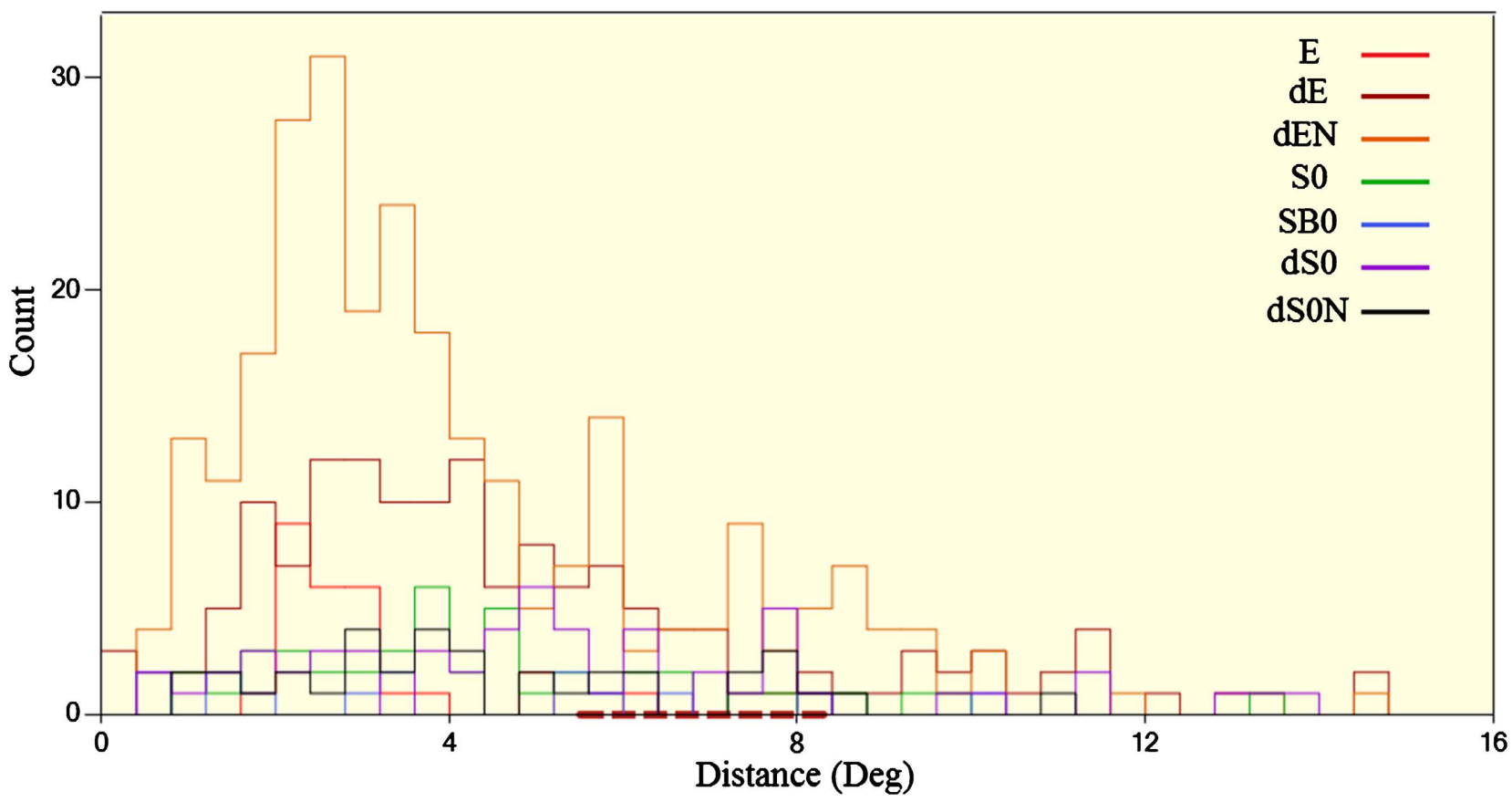

Figure 15. Distribution of ETGs by type.

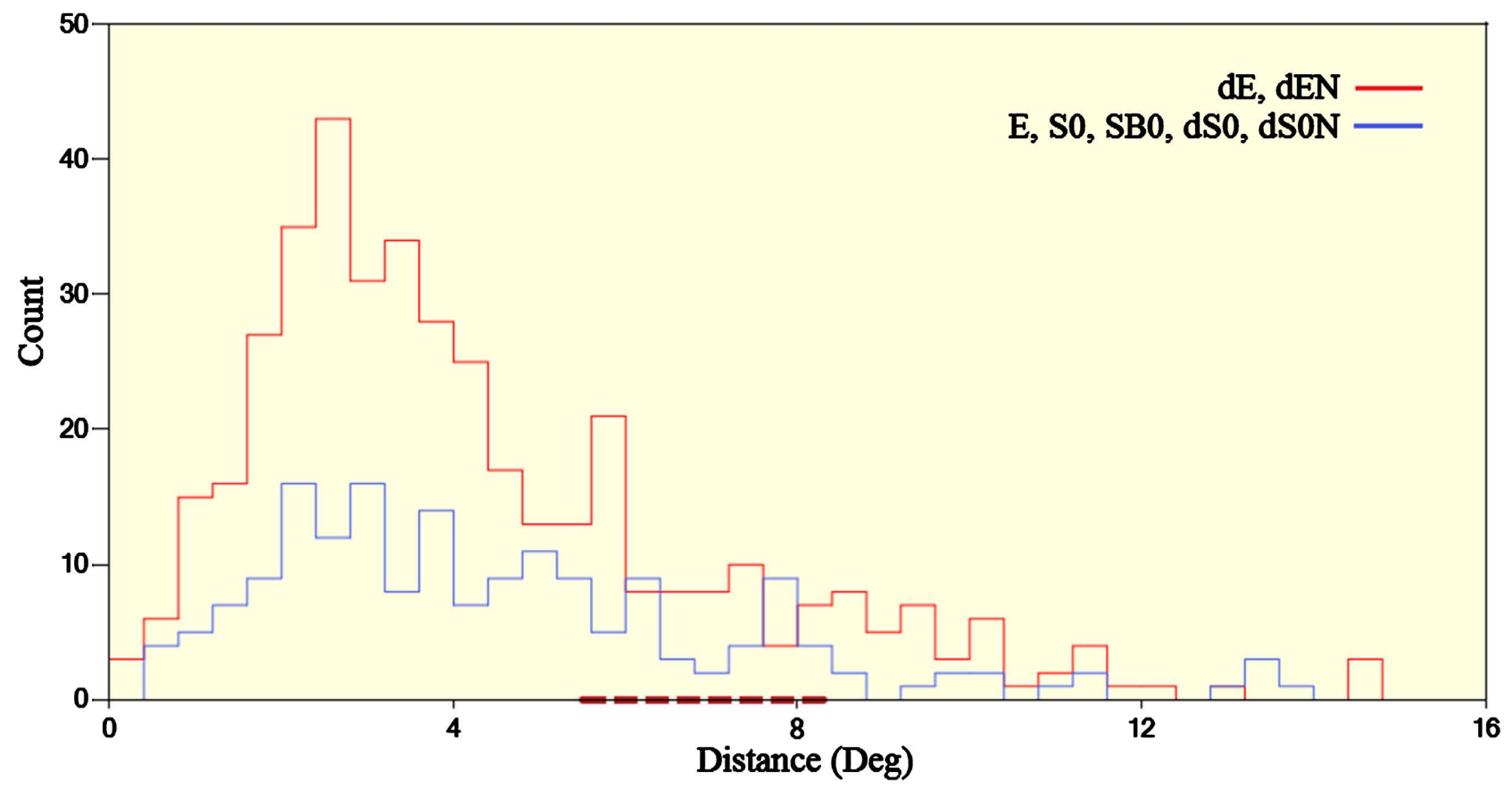

Figure 16. Distribution of ETG groupings.

what one would expect from the capture of a random assortment of LTGs that came into existence outside the cluster. Again, we show the uniform density curve and it is a good match to the LTG distributions.

We will next consider the radial velocities. In Figure 19, we show the radial velocities organized by galaxy type.

From the figure, we see that the distribution of velocities is, with two exceptions, 


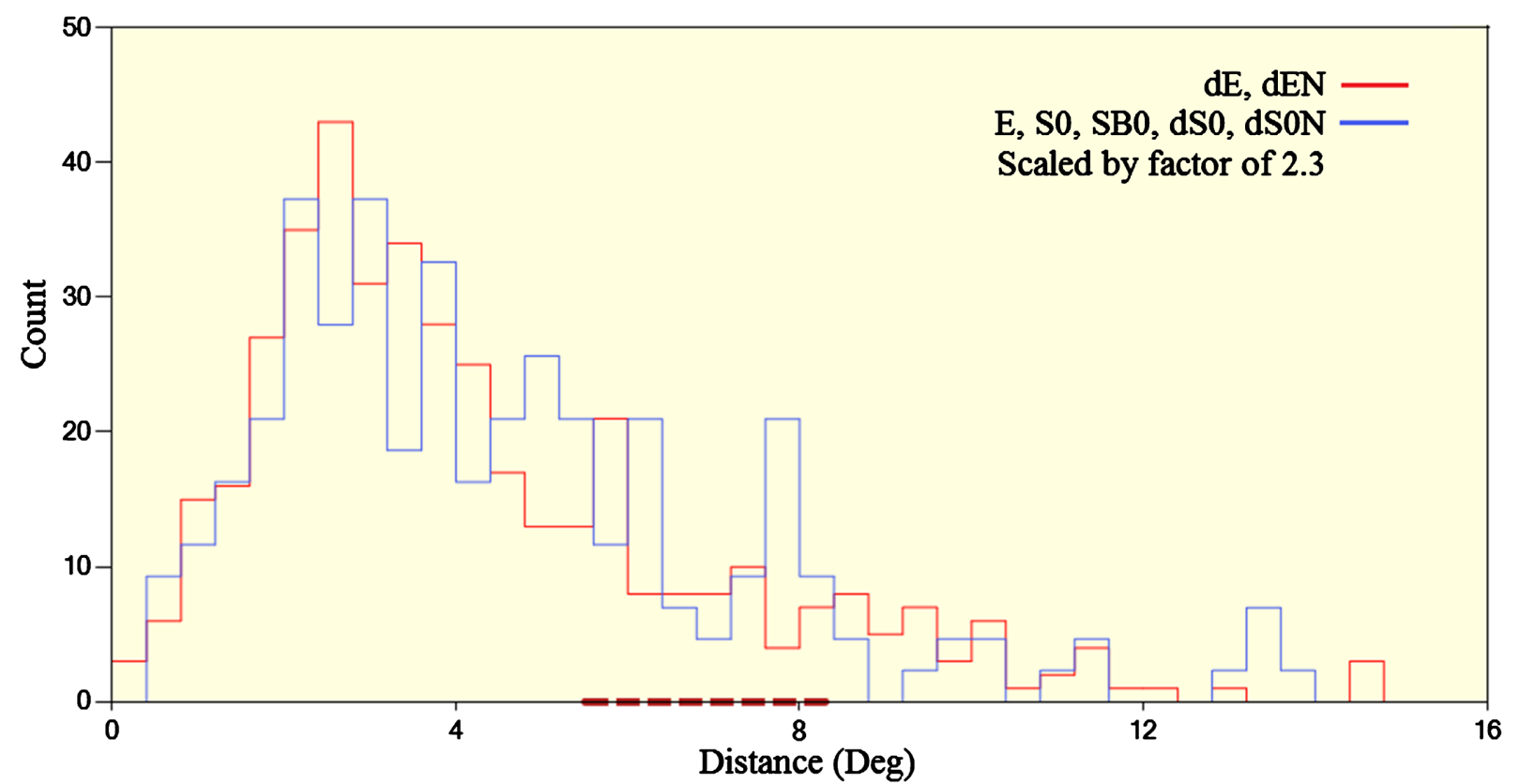

Figure 17. Distribution of ETG groupings with the E, etc. grouping scaled by the ratio of the actual counts of galaxies.

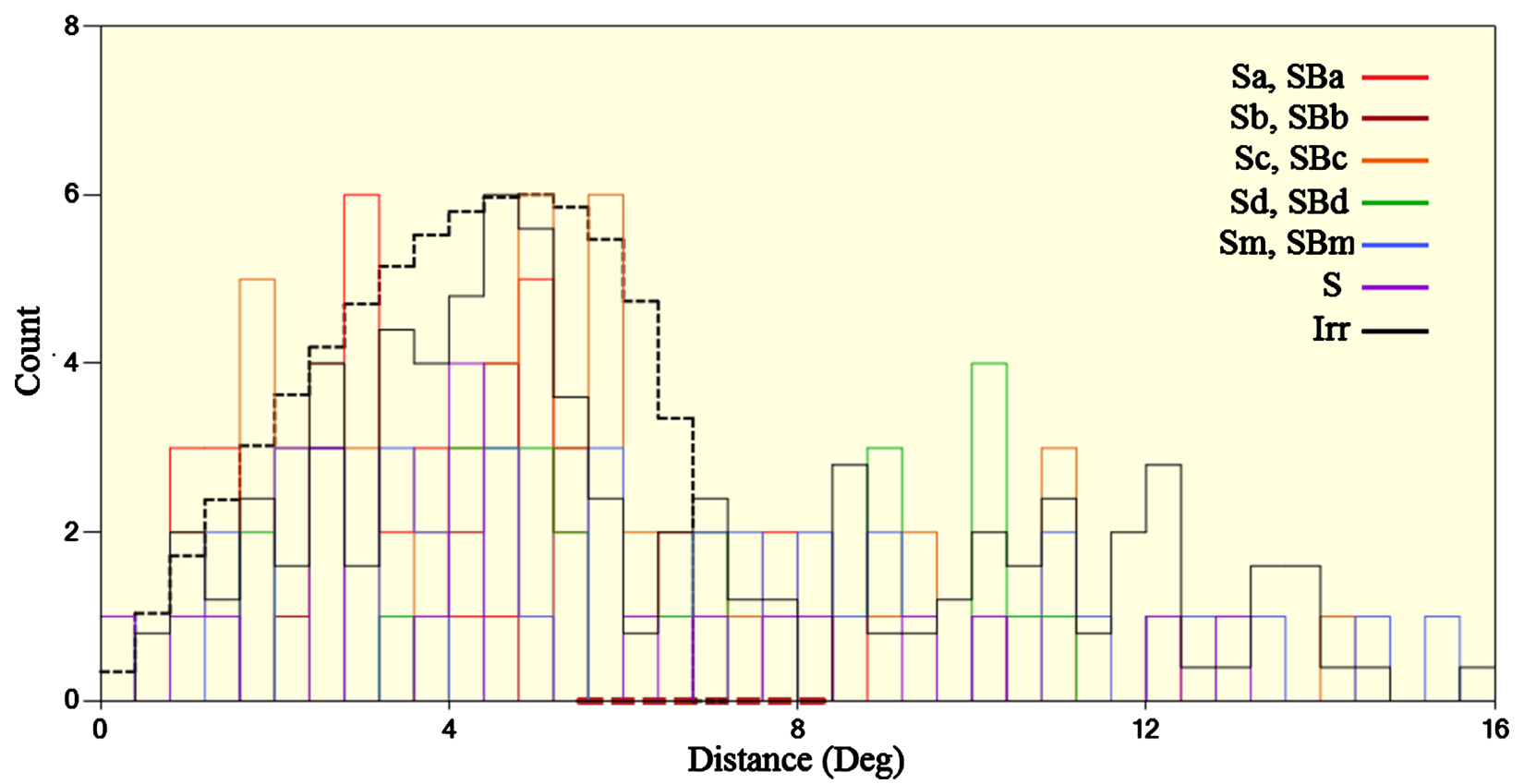

Figure 18. Distribution of LTGs by type. The count of the irregulars is nearly equal to the sum of all the LTGs combined. To compensate for this, their normalization was adjusted to match that of the others.

more or less the same for all galaxy types. We also see that the averages are generally below the center of the Hubble flow band which is a consequence of the fact that the Local Group is approaching the Virgo Cluster at a rate between 100 $400 \mathrm{~km} \cdot \mathrm{s}^{-1}$. The exceptions are the averages for the Sb and SBd types which both show noticeable dips. The dip for Sb, which was noticed by H. Arp [9]. is probably just a consequence of the small sample. If the $\mathrm{Sb}$ are combined with the $\mathrm{SBb}$, the dip largely disappears. The same is true of the SBd dip if they are combined 
with Sd. Arp also reported higher than average velocities for Sc and others but that is not seen in the results shown here.

We have been ignoring the possibles but just to show that they do form a distinct group, in Figure 20, we show their velocity distributions.

We see indeed, that their distribution is quite different.

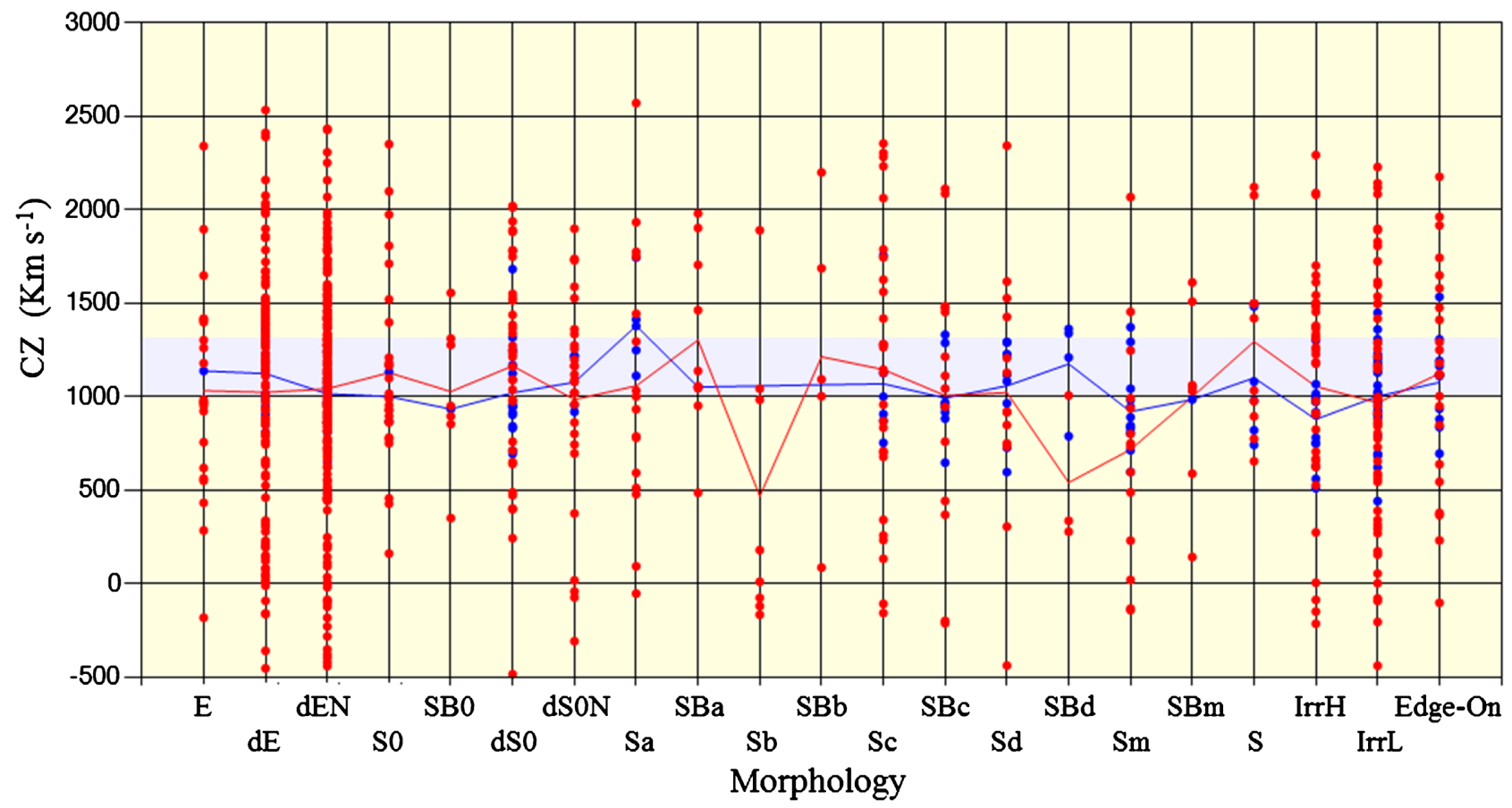

Figure 19. Radial velocities by galaxy type. The blue band represents the Hubble flow for a Hubble parameter of $70 \mathrm{~km} \cdot \mathrm{s}^{-1} \cdot \mathrm{Mpc}^{-1}$. The width of the band corresponds to the diameter of the cluster assuming a spherical geometry for the cluster.

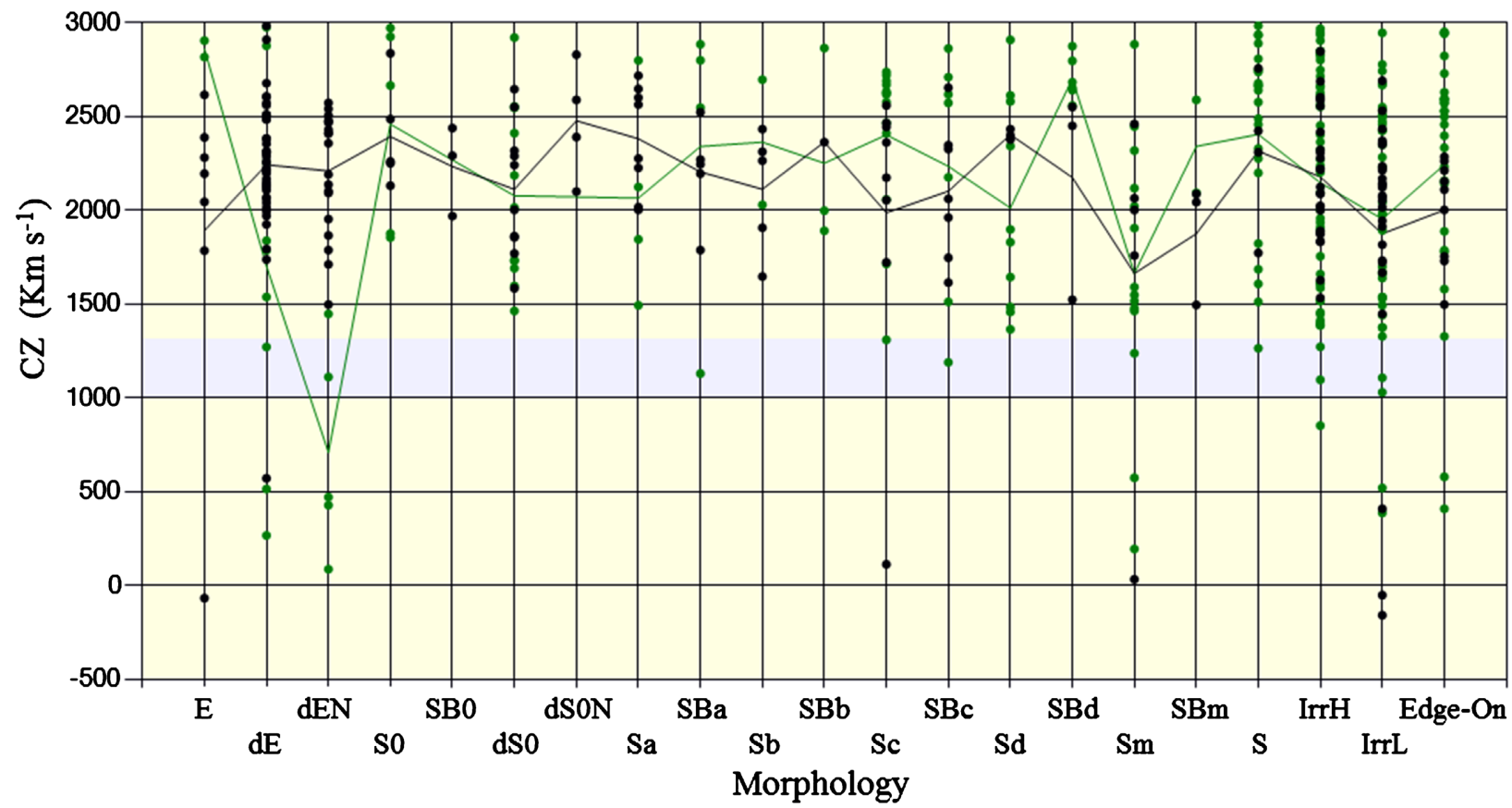

Figure 20. Radial velocities by galaxy type of the EVCC possibles. 
Figure 21 displays the distribution of velocities across the cluster. The galaxies were sorted by dividing the range of velocities in the catalogue into 6 bins and then assigning a different color to each bin.

Figure 22 gives another point of view on the same data. (The combination of
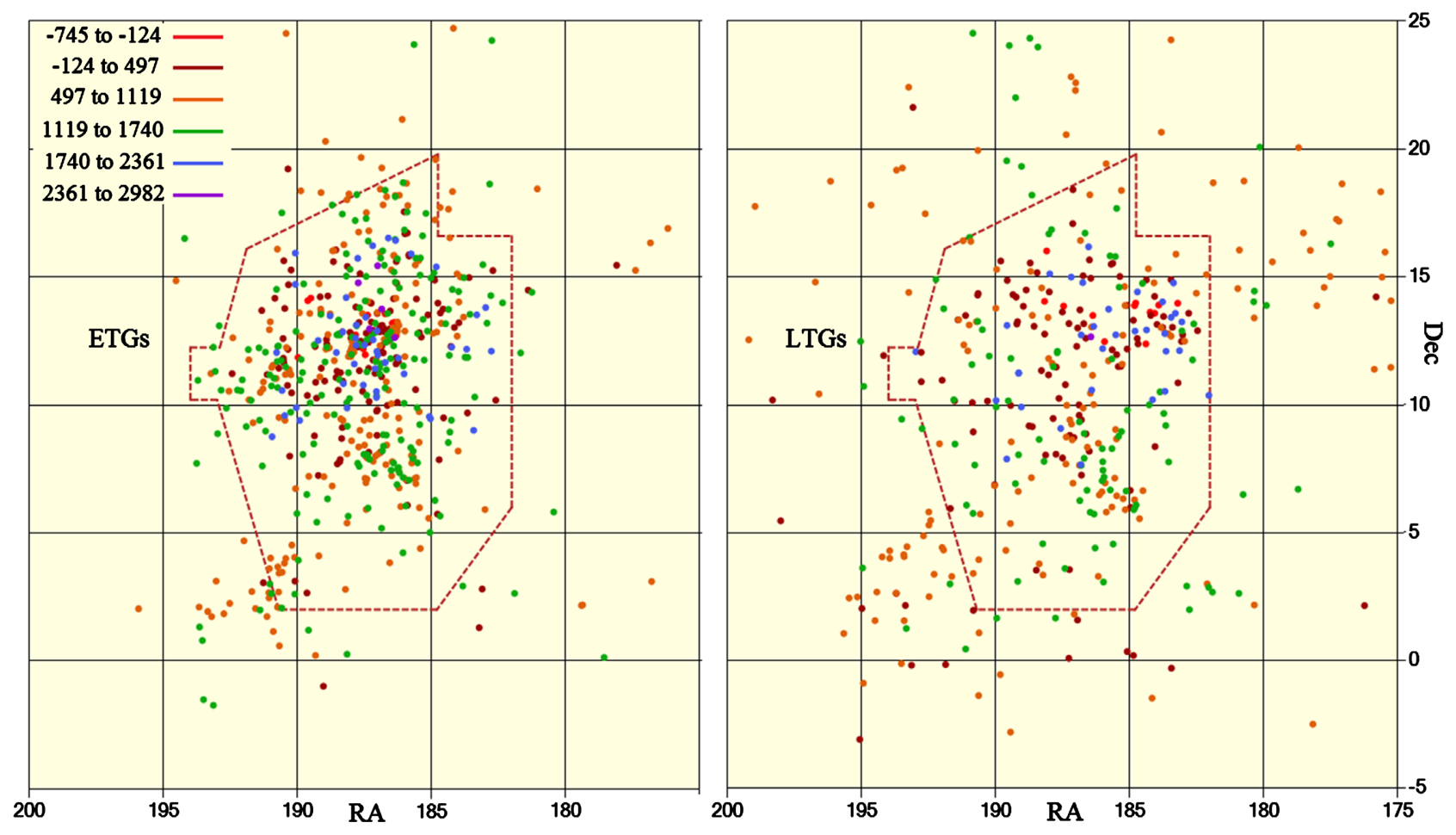

Figure 21. Distribution of velocities by position.
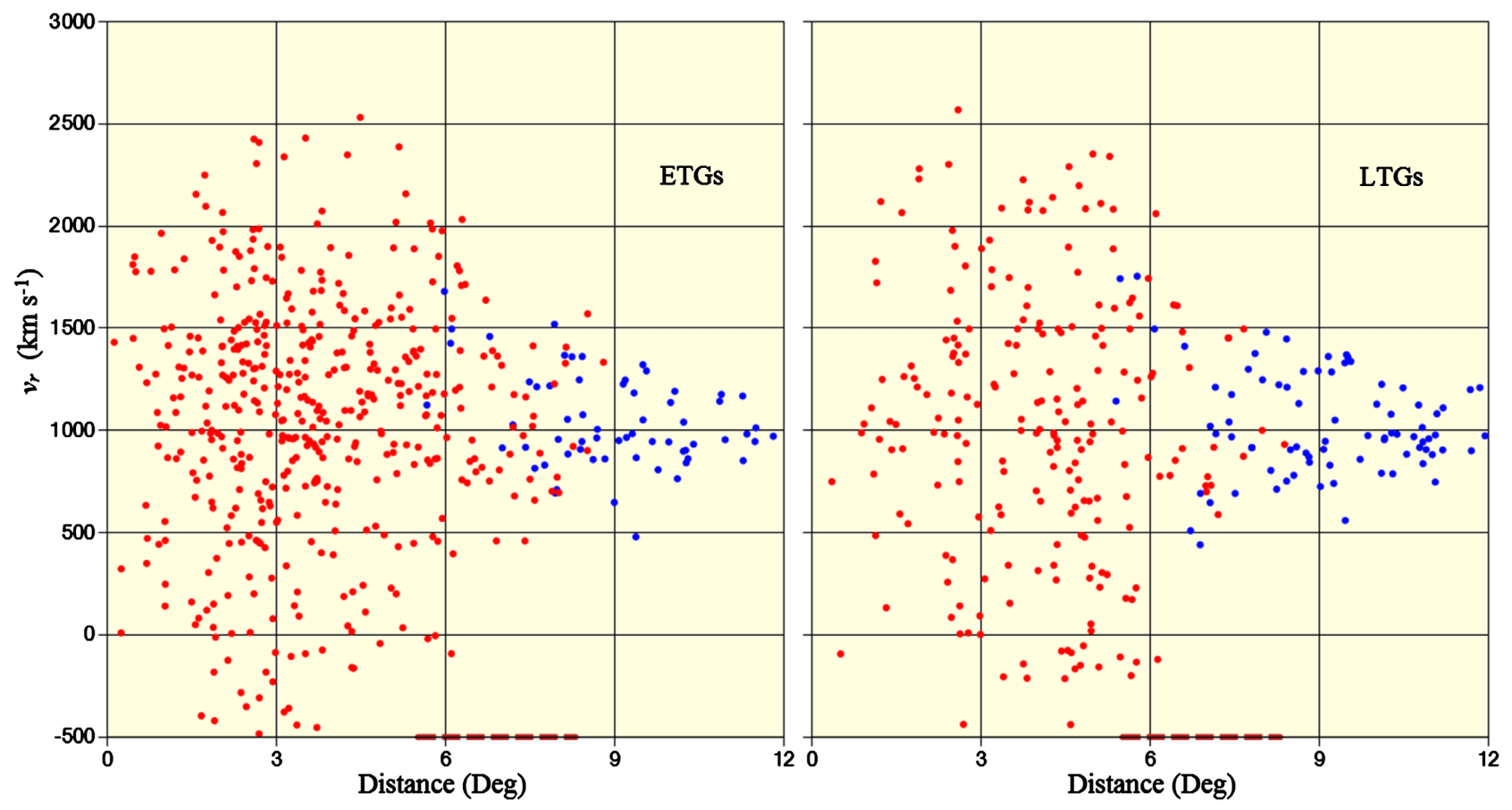

Figure 22. Distribution of velocities by distance. Red and blue denote galaxies inside and outside the boundary respectively. 
these two data sets is that used in Figure 10.)

Outside the cluster boundary, the velocities are close to the Hubble flow values, and inside, the range increases towards the center with no obvious bias that would indicate a significant rotation. There is also no obvious difference between the ETGs and LTGs distributions. This indicates that all the galaxies for the most part acquired their velocities from their common interaction with the mass of the cluster. This is also seen in the similarity between the paths of the internal and external galaxies in Figure 5.

To summarize, we find good evidence that there are two distinct populations and that the ETG population originated inside the cluster. The distinct distributions also indicate that the ETGs originated as such and are not the result of a wholesale morphological change from some initial population of LTGs. If the latter were the case, then one would expect a mix of ETGs and LTGs instead of all ETGs since no conversion process would be $100 \%$ effective. Galaxies that crossed the boundary from outside the cluster are primarily LTGs which is what one would expect since LTGs dominate the distributions outside of clusters.

What we are finding is that the imprints that code for clusters also code for just ETGs inside those clusters. The why of this we won't know until we discover how the Plank era inflation worked.

Having dealt with galaxy clusters, we will now step down to the galaxies.

\section{Galaxies}

We will now apply the same model to the formation of galaxies. Galaxies are much denser than the ICM so the model parameters will be quite different. Assuming a background density of $1 \mathrm{~m}^{-3}$ and, using the $\mathrm{MW}$ as our example, an average present-day density of $2.7 \times 10^{6} \mathrm{~m}^{-3}$, we obtain an outer surface value of $f_{\text {sur }}=140$. We perform the calculation for several values of $f_{s}$ with the results shown in Figure 23.

We find that a value of $f_{s}=55$ results in the zero-velocity point coinciding with $t_{G}$. An interesting point is that the model predicts that our sample galaxy reached $t_{G}$ with a size considerably larger than its final size. This behavior seems

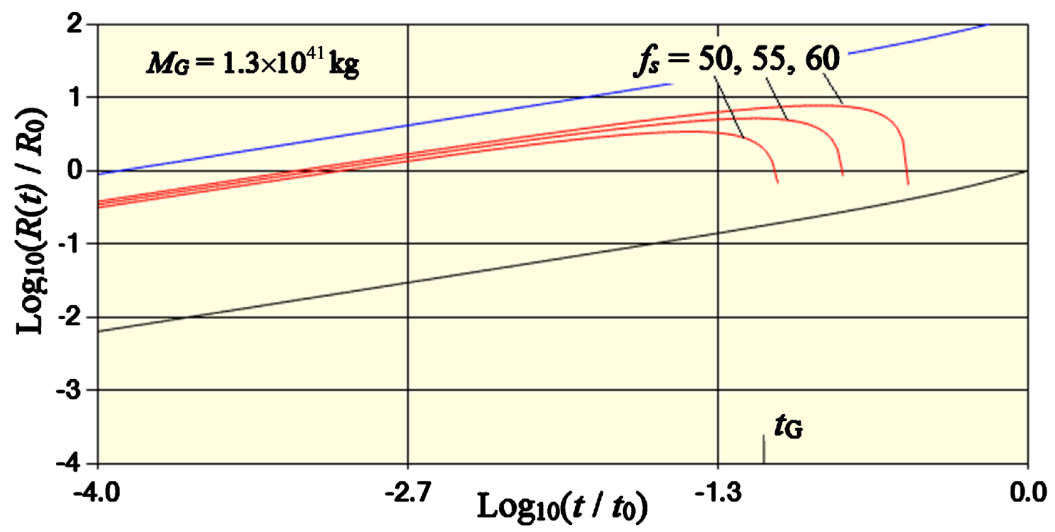

Figure 23. Galaxy model calculation for three values of $f_{s}$. 
to be general over a wide range of galactic sizes and masses and was a critically important factor leading to the formation of the large $\mathrm{H}_{\mathrm{I}}$ rings. The size ratio does vary with the size and mass of the galaxy. In this case, with $f_{s}=55$, the galaxy reached a maximum size of $5.2 R_{0}$ which is in reasonable agreement with a compaction factor of 4 that we found during our study of the $\mathrm{H}_{\mathrm{I}}$ rings. This will be discussed in Part 2. It follows from this that there must have been a subsequent contraction which is illustrated in Figure 24.

The curve is not the result of a model calculation but just a hand-drawn curve to illustrates the idea. The actual calculation would require solving the complete set of Einstein's equations for a galaxy and that is beyond the scope of the paper.

With $f_{s}=55$, the initial size of the MW would have been about $2.75 \times 10^{6} \mathrm{ly}$ in present-day terms which is about the present-day distance to the Andromeda galaxy. The proton density at the time, $t=4000 \mathrm{~s}$, would have been about 17 $\mathrm{m}^{-3}$ in present-day terms.

Referring now to the vacuum energy density, assuming it underwent the same compaction as the particles, with a total compaction of 140 , the present-day density would be on the order of $5.5 \times 10^{-4} \mathrm{j} \cdot \mathrm{m}^{-3}$. Dividing by the mass-energy of a proton, this yields an equivalent particle density of $3.7 \times 10^{6} \mathrm{~m}^{-3}$ which is close to the average density of the galaxy again lending support to the idea that vacuum energy is the reality of dark matter.

Earlier we proposed that galaxy rotation is, to a great extent, a matter of the rotation of the vacuum. The results of this section suggest that the vacuum rotation largely accounted for the rotation at the ZVP, which as will see in Part 2, explains the rotation of the large $\mathrm{H}_{\mathrm{I}}$ rings and their flat velocity distribution. Subsequently, the rotation of the matter would have increased in the usual way as the galaxies underwent contraction.

For comparison, we will finish this section with the solution for a representative dwarf galaxy. Using a mean radius of $R_{0}=1.1 \times 10^{19} \mathrm{~m}$ and a mean mass of $1.1 \times 10^{39} \mathrm{~kg}$ and assuming a disk-shaped morphology, we find a mean density of $1.2 \times 10^{9} \mathrm{~m}^{-3}$. Comparing with the average density of the MW, we see that dwarf galaxies are much denser. These values result in a value of $f_{\text {Sur }}=1057$. The

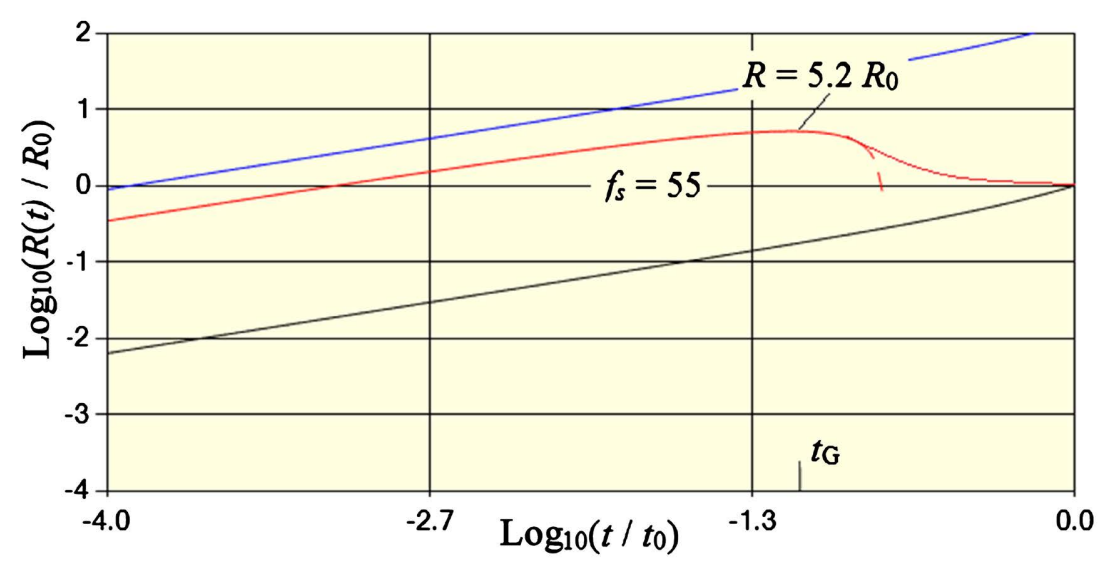

Figure 24. Possible galaxy evolution after passing the zero-velocity point. 
result is shown in Figure 25.

We find a result similar to that of the MW but with a considerably larger zero-velocity size. In this case, the ratio is 45.9 as opposed to a value of 5.2 for the MW. These sizes are relative to the final size of the galaxy in each case. The ratio of the present-day sizes of the MW and our dwarf galaxy is 43 but the ratio at the time of nucleosynthesis was only 4.9 .

Lastly, we did consider the possibility that galaxies formed inside a cluster might have different properties than those formed outside. Those formed inside would have had an initial background particle density of $2.5 \mathrm{~m}^{-3}$ instead of $1 \mathrm{~m}^{-3}$ but the resulting differences were very small so there is no distinction between the two sets in terms of place of origin. As noted earlier, the predominance of ETGs in general, and $\mathrm{dE}$ and $\mathrm{dEN}$ galaxies in particular, is a consequence of the imprint and not the mechanics of galaxy formation.

\section{Stars}

We next move on to the stars using the Sun as our example. Although stars are extremely dense compared to everything else, that high density arises as a consequence of their gravitational collapse. At the time of nucleosynthesis, on the other hand, we will find that their density was essentially the same as that of their host galaxy. Their present-day size is also not representative of the situation at the time of nucleosynthesis so instead of dealing with dimensions characteristic of a collapsed star, we will consider the problem of the evolution of a gas cloud with a nominal present-day dimension of 1 ly which happens to be the size of the Oort cloud. All the stars had to pass through that dimension during their evolution so it is a reasonable assumption. It turns out that the results are not sensitive to the exact value used in any case.

We discussed earlier the immense volume of space that would be required to build a star out of the density of the $1 \mathrm{~m}^{-3}$ background. Stars, however, don't start from the empty background but from the average density of their host galaxy which for the MW was $17 \mathrm{~m}^{-3}$. That still doesn't eliminate a huge volume requirement but it helps a little. We have then,

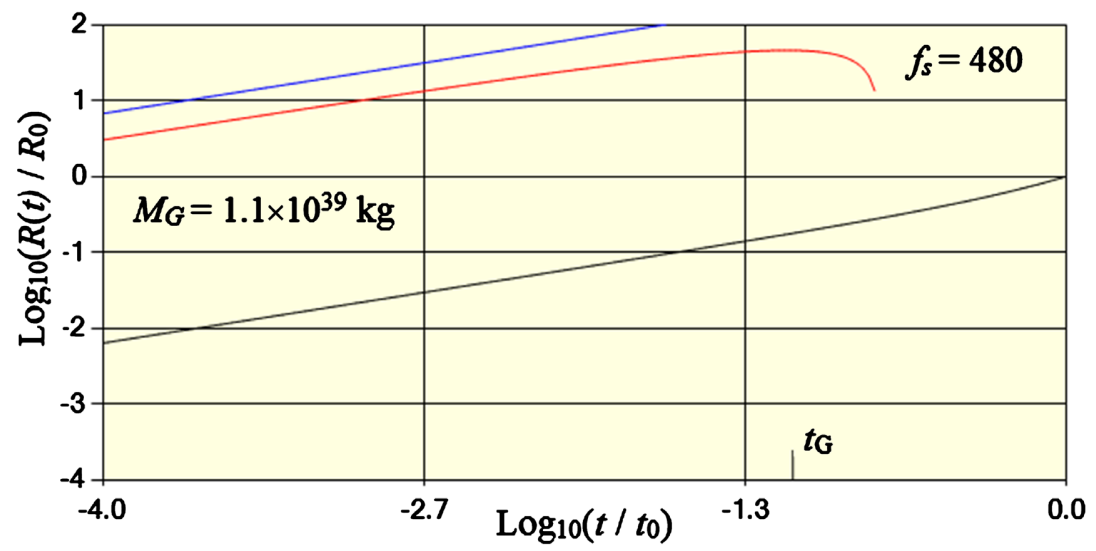

Figure 25. Model calculation for a dwarf galaxy. 


$$
\frac{4 \pi}{3} R_{*}^{3} m_{\text {proton }} \times 17=2 \times 10^{30} .
$$

Solving for the radius, we find $R_{*}=271 \mathrm{ly}$ and since our nominal size is $1 \mathrm{ly}$, we have $f_{\text {Sur }}=271$.

In Figure 26, we show the results for a range of $f_{s}$ values.

It now looks like we have a problem. The model seems to allow for star formation going back in time as far as one likes because, unlike galaxies and clusters, the ultimate size of the star is not the nominal value we have used. This, however, is not the case and the critical element is the temperature of the gas. From Jean's model of star formation, we have a simple criterion that must be satisfied before a gas cloud can collapse into a star.

$$
P E+K E<0
$$

If the kinetic energy is too large, no collapse can occur. In this case,

$$
P E=-\frac{3}{5} \frac{G M^{2}}{R}
$$

and

$$
K E=N_{p} \frac{3}{2} k_{b} T_{c}
$$

where $N_{p}$ is the total number of protons in the cloud. Solving for the critical temperature we find,

$$
T_{c}=\frac{0.683}{R(\mathrm{ly})} \mathrm{K}
$$

where $R$ is the radius of the cloud at the point of zero-velocity measured in $l y$.

We now consider the actual temperature of the gas. At the time of recombination, the temperature of the $\mathrm{CMB}$ was on the order of $4000 \mathrm{~K}$ and the gas cloud would have been in thermal equilibrium with the CMB. After that time, the protons would have bounded with electrons and disconnected from the CMB. It is reasonable to assume that they henceforth behaved as an ideal gas and since there did not yet exist any energy sources, the subsequent expansion would have

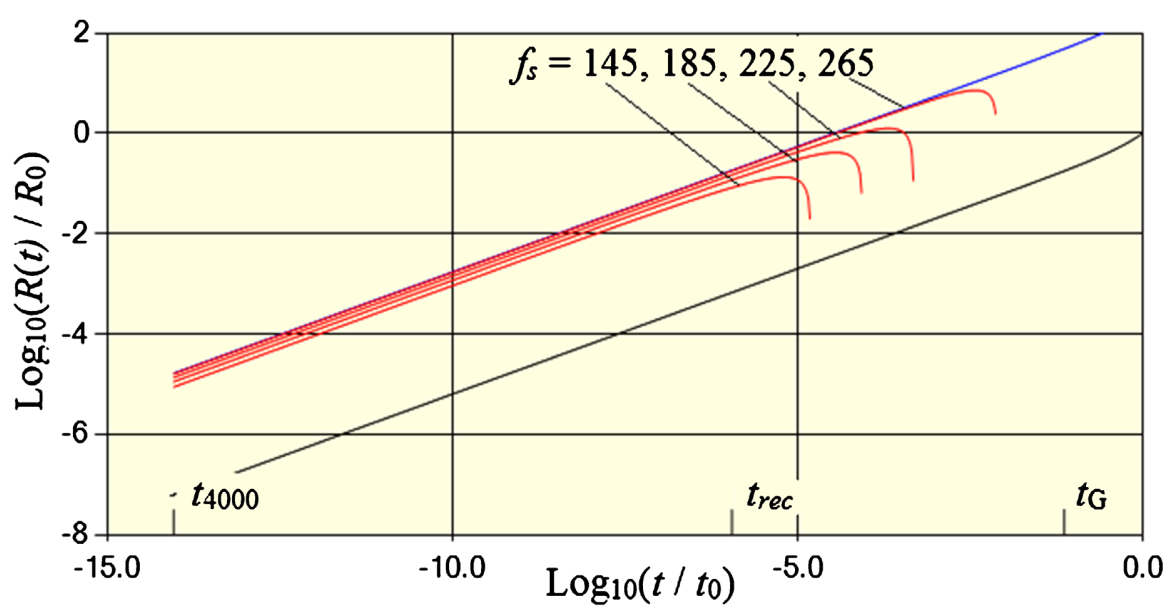

Figure 26. Model solution for a star with a range of $f_{s}$ values. 
been adiabatic. The temperature would then have varied as $T \propto V^{-2 / 3}$ or $T \propto l(t)^{-2}$ where $l(t)$ is a time-dependent dimension characterizing the volume of the gas. Thus, we have

$$
T_{\text {gas }}(t)=4000\left(\frac{a\left(t_{\text {rec }}\right)}{a(t)}\right)^{2} \mathrm{~K}
$$

In Figure 27, we show the two curves.

We find that the actual temperature is greater than the critical temperature over the whole range. It appears that that remains true even at $t=t_{G}$ but that is a consequence of the log-log plot. The values at $t_{G}$ are quite small, 0.058 vs. 0.019 so the distinction is meaningless. Referring back to the cases shown in Figure 26, the actual gas temperature is too large for any compaction to occur. Instead, the gas continued to expand as a result of the scaling until $t \approx t_{G}$ when the collapse could begin. The conclusion is that, even though each star began with a single $f_{s}$ value, any value from a wide range of possible values would have worked. We also see that the compaction of the earliest stars could have happened before the zero-velocity point of their host galaxy.

We now ask, what $f_{s}$ value results in the ZVP occurring at $t_{G}$ ? The answer is that it must be very close to the $f_{\text {Sur }}$. Figure 28 shows the result with $f_{s}=270.9$.

It is unlikely that such a small compaction could be realized over the required huge volume so the evolutionary paths with larger initial compaction probably describe reality.

Finally, based on just their initial and final sizes, for the MW, the ratio of the volume of the galaxy to that of a star would allow for more than $10^{12}$ stars at the time of nucleosynthesis and $4 \times 10^{11}$ stars at $t=t_{G}$. The mass of the MW, however, is about $6.5 \times 10^{10} M_{\odot}$ which sets an upper limit on the number of stars of a size $M_{\odot}$ that could have been created initially. Of course, the actual number could have been considerably smaller. The point is that there was no volume constraint on the star creation process.

In conclusion, we see that the model can account for the creation of stars, and

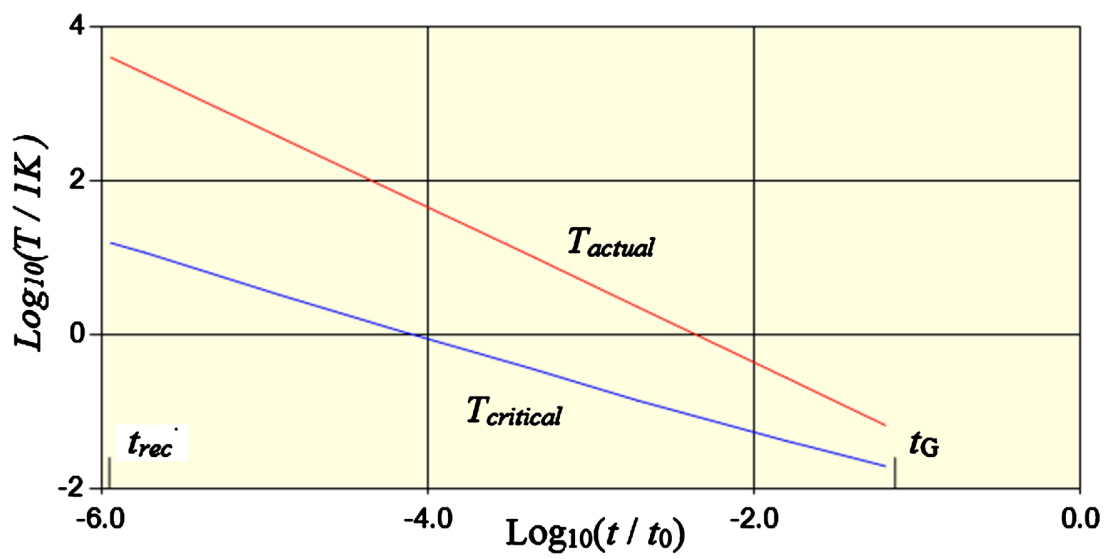

Figure 27. Comparison of the actual and critical temperatures. 
further, it places their creation at a time no earlier than at $t=t_{G}$.

\section{The Rule}

In the preceding, we found that by adjusting the value of $f_{s}$, we could arrange for the ZVP for any structure to occurs at the time, $t=t_{G}$. The imprint, however, can't follow our procedure of looking ahead at the outcome and then adjusting $f_{s}$ to achieve the desired result. That being the case, there must have been a property built into the vacuum imprint that predetermined the correct value to use at the time of nucleosynthesis. Recall that $f_{s} R_{0}$ gives the initial size of any structure. In Figure 29, we show a plot of this size, adjusted for the expansion, as a function of the mass of each structure.

We find that the initial size of each structure had a power law relationship with its mass given by

$$
f_{s} R_{0}=2.06 \times 10^{4} \mathrm{~m}^{0.28} .
$$

In [1], we found a similar power-law relationship between the present-day size of structures and their mass, namely $R_{0} \propto m^{0.68}$. Combining, we find that

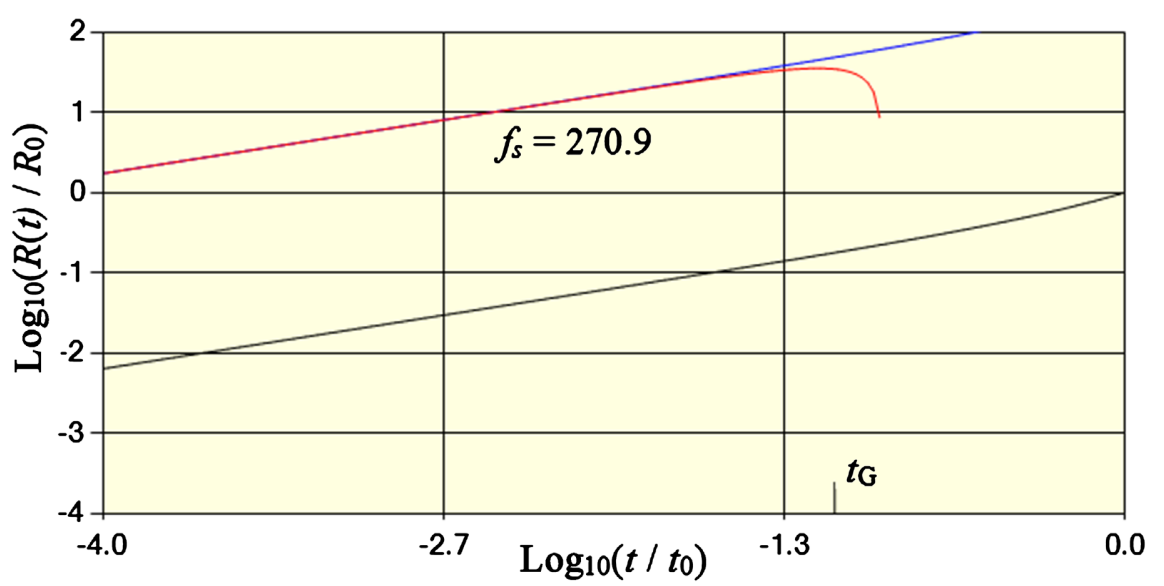

Figure 28. Model result for a star with initial compaction of 1.0004 .

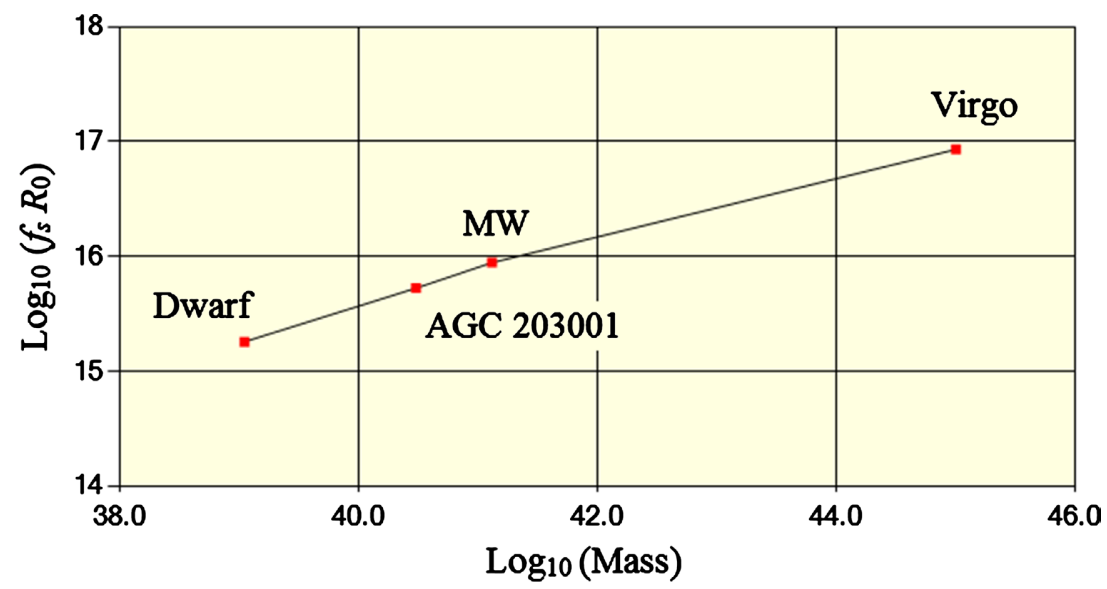

Figure 29. Initial size vs mass relationship. The galaxy ACC 203001 is discussed in Part 2. It is a lenticular galaxy roughly the same size as the MW but with a smaller mass. 
$f_{s} \propto m^{-0.4}$.

This rule does not, however, work for stars but as we saw in the previous section, the time of their collapse is determined by the temperature of the gas rather than by their nominal ZVP.

\section{The Origin of HI Rings}

At the time of the ZVP, all galaxies consisted of nothing more than gas clouds with the important attribute that they were several times larger than the final size of the galaxies so compaction began after the ZVP. The origin of the larger outer rings can now be stated. They are the outer regions of some galaxies that remained at the galaxies' maximal size instead of contracting with the inner regions as they compacted to their final size. The range of sizes of the rings relative to the present-day sizes of their host galaxies is easily understood because the new model predicts just such a range of maximal galaxy sizes. We gave two such examples earlier. The Milky Way (MW) had a ZVP size equal to 5.2 times its present-day size and a hypothetical dwarf galaxy had a ZVP size 45.9 times its present-day size.

As discussed in Part 2, there is ample observational evidence showing that the outer rings are kinematically disconnected from the compacted host galaxy which supports this idea and the principal remaining problem is to understand how this disconnection came about. LTGs do not have larger outer rings whereas about $50 \%$ of ETGs do have such rings. This indicates that is the initial mass distribution of the proton gas that is the deciding factor. In Part 2, we discuss the whole HI structure problem in detail.

\section{Conclusions}

In this paper, we have shown that the new model of cosmology can account for the initial formation of all cosmic structures ranging in size from stars up to superclusters. Along the way, we have presented a model that can account for the energy density of the ICM and have shown that at the time of galaxy formation, galaxies had sizes considerably larger than their present-day sizes. We have also shown that vacuum energy can account for the phenomena attributed to dark matter.

In Part 2 of this work, we will show that the origin and evolution of $\mathrm{H}_{\mathrm{I}}$ rings can be readily understood in terms of the structure evolutions describe in this paper.

\section{Conflicts of Interest}

The author declares no conflicts of interest regarding the publication of this paper.

\section{References}

[1] Botke, J.C. (2020) A Different Cosmology-Thoughts from Outside the Box. Jour- 
nal of High Energy Physics, Gravitation and Cosmology, 6, 473-566.

https://doi.org/10.4236/jhepgc.2020.63037

[2] van Driel, W. and van Woerden, H. (1991) Distribution and Motions of Atomic Hydrogen in Lenticular Galaxies XI. A Summary of HI Observations and Evolutionary Scenarios. Astronomy and Astrophysics, 243, 71-92.

[3] Oesch, P.A., et al. (2016) A Remarkably Luminous Galaxy at Z = 11.1 Measured with Hubble Space Telescope Grism Spectroscopy. ArXiv: 1603.00461v1.

[4] Manolopoulou, M. and Plionis, M. (2017) Galaxy Cluster's Rotation. Monthly Notices of the Royal Astronomical Society, 465, 2616-2633.

https://doi.org/10.1093/mnras/stw2870

[5] Wikipedia (2021) Intracluster medium. https://en.wikipedia.org/wiki/Intracluster_medium

[6] Suk, K., et al. (2014) The Extended Virgo Cluster Catalog, ArXiv: 1409.3283v1.

[7] Binggeli, B., Sandage, A. and Tammann, G.A. (1985) Studies of the Virgo Cluster. II. A Catalog of 2096 Galaxies in the Virgo Cluster Area. Astronomical Journal, 90, 1681-1758. https://doi.org/10.1086/113874

[8] Serra, P., et al. (2012) The ATLAS Project-XIII. Mass and Morphology of HI in Early-Type Galaxies as a Function of Environment. Monthly Notices of the Royal Astronomical Society, 422, 1835-1862.

https://doi.org/10.1111/j.1365-2966.2012.20219.x

[9] Arp, H. (1988) What Is the Mean Redshift of the Virgo Cluster? Astronomy \& Astrophysics, 202, 70-76. 